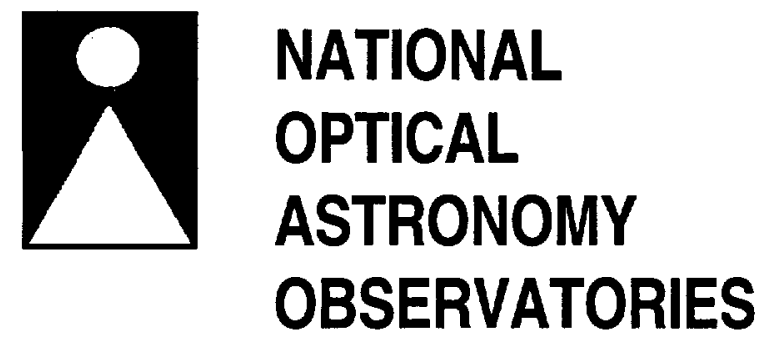

Preprint Series

NOAO Preprint No. 815

Standard Giant Branches in the Washington Photometric System

Doug Geisler

Ata Sarajedini

To be published in: Astronomical Journal,

January 1999

October 1998

Operated for the National Science Foundation by the Association of Universities for Research in Astronomy, Inc. 



\title{
Standard Giant Branches in the Washington Photometric System
}

\author{
Doug Geisler ${ }^{1}$ \\ Kitt Peak National Observatory, National Optical Astronomy Observatories, \\ P.O. Box 26732, Tucson, Arizona 85726 \\ dgeisler@noao.edu \\ and \\ Ata Sarajedini ${ }^{2}$ \\ San Francisco State University, Dept. of Physics and Astronomy, \\ 1600 Holloway Ave., San Francisco, CA 94132 \\ ata@stars.sfsu.edu
}

Received —

astro-ph/9809393; to be published in January 1999 AJ

${ }^{1}$ Visiting Astronomer, Canada-France-Hawaii Telescope, operated by the National Research Council of Canada, le Centre National de la Recherche Scientifique de France, and the University of Hawaii.

${ }^{2}$ Hubble Fellow 



\begin{abstract}
We have obtained CCD photometry in the Washington system $C, T_{1}$ filters for some 850,000 objects associated with 10 Galactic globular clusters and 2 old open clusters. These clusters have well-known metal abundances, spanning a metallicity range of 2.5 dex from $[\mathrm{Fe} / \mathrm{H}] \sim-2.25$ to +0.25 at a spacing of $\sim 0.2$ dex. Two independent observations were obtained for each cluster and internal checks, as well as external comparisons with existing photoelectric photometry , indicate that the final colors and magnitudes have overall uncertainties of $\lesssim$ $0.03 \mathrm{mag}$.
\end{abstract}

Analogous to the method employed by Da Costa and Armandroff (1990, AJ, 100,162 ) for $V, I$ photometry, we then proceed to construct standard ( $M_{T_{1}}$ , $\left.\left(C-T_{1}\right)_{0}\right)$ giant branches for these clusters adopting the Lee et al. (1990, ApJ, 350, 155) distance scale, using some 350 stars per globular cluster to define the giant branch. We then determine the metallicity sensitivity of the $\left(C-T_{1}\right)_{0}$ color at a given $M_{T_{1}}$ value. The Washington system technique is found to have three times the metallicity sensitivity of the $V, I$ technique. At $M_{T_{1}}=-2$ (about a magnitude below the tip of the giant branch, roughly equivalent to $M_{I}=-3$ ), the giant branches of 47 Tuc and M15 are separated by 1.16 magnitudes in $\left(C-T_{1}\right)_{0}$ and only 0.38 magnitudes in $(V-I)_{0}$. Thus, for a given photometric accuracy, metallicities can be determined three times more precisely with the Washington technique. We find a linear relationship between $\left(C-T_{1}\right)_{0} \quad$ (at $M_{T_{1}}=-2$ ) and metallicity (on the Zinn 1985, ApJ, 293,424 scale) exists over the full metallicity range, with an $\mathrm{rms}$ of only 0.04 dex. We also derive metallicity calibrations for $M_{T_{1}}=-2.5$ and -1.5 , as well as for two other metallicity scales. The Washington technique retains almost the same metallicity sensitivity at faint magnitudes, and indeed the standard 
giant branches are still well separated even below the horizontal branch. The photometry is used to set upper limits in the range $0.03-0.09$ dex for any intrinsic metallicity dispersion in the calibrating clusters. The calibrations are applicable to objects with ages $\gtrsim 5$ Gyr - any age effects are small or negligible for such objects.

This new technique is found to have many advantages over the old two-color diagram technique for deriving metallicities from Washington photometry - In addition to only requiring 2 filters instead of 3 or 4 , the new technique is generally much less sensitive to reddening and photometric errors, and the metallicity sensitivity is many times higher. The new technique is especially advantageous for metal-poor objects. The five metal-poor clusters determined by Geisler et al. (1992, AJ, 104, 627), using the old technique, to be much more metal-poor than previous indications, yield metallicities using the new technique which are in excellent agreement with the Zinn scale. The anomalously low metallicities derived previously are undoubtedly a result of the reduced metallicity sensitivity of the old technique at low abundance. However, the old technique is still competitive for metal-rich objects $([\mathrm{Fe} / \mathrm{H}]$ $\gtrsim-1)$

We have extended the method developed by Sarajedini (1994, AJ, 107, 618) to derive simultaneous reddening and metallicity determinations from the shape of the red giant branch, the $T_{1}$ magnitude of the horizontal branch, and the apparent $\left(C-T_{1}\right)$ color of the red giant branch at the level of the horizontal branch. This technique allows us to measure reddening to 0.025 magnitudes in $E(B-V)$ and metallicity to 0.15 dex. Reddenings can also be derived from the blue edge of the instability strip, with a similar error.

We measure the apparent $T_{1}$ magnitude of the red giant branch bump 
in each of the calibrating clusters and find that the difference in magnitude between the bump and the horizontal branch is tightly and sensitively correlated with metallicity, with an rms dispersion of 0.1 dex. This feature can therefore also be used to derive metallicity in suitable objects. Metallicity can be determined as well from the slope of the RGB, to a similar accuracy. Our very populous color- magnitude diagrams reveal the asymptotic giant branch bump in several clusters.

Although $M_{T_{1}}$ of the red giant branch tip is not as constant with metallicity and age as $M_{I}$, it is still found to be a useful distance indicator for objects with $[\mathrm{Fe} / \mathrm{H}] \lesssim-1.2$. For the 6 standard clusters in this regime, $<M_{T_{1}}(T R G B)>=-3.22 \pm 0.11(\sigma)$, with only a small metallicity dependence. This result is found to be in very good agreement with the predictions of the Bertelli et al. (1994, A\&AS, 106, 275) isochrones. We also note that the Washington system holds great potential for deriving accurate ages as well as metallicities .

Subject headings: Galaxy: abundances - globular clusters: general 



\section{INTRODUCTION}

The color of the first ascent, red giant branch (RGB) of an old stellar system has long been recognized as a sensitive indicator of metal abundance (e.g. Sandage and Smith 1966, Hartwick 1968, Rood 1978, Frogel et al. 1983). Observationally, the utility of this feature for determining metallicity was first exploited using the traditional Johnson BV filters by Hartwick (1968) in his definition of the $(B-V)_{0, g}$ feature: the reddening -corrected color of the RGB at the level of the horizontal branch (HB). Searle and Zinn (1978) formed an abundance ranking from plots of $M_{V}$ vs. a reddening-independent color derived from spectral scans of globular cluster (GC) giants.

Da Costa and Armandroff (1990, hereafter DCA) extended this technique to the $V, I$ (Cousins) photometric system, quoting a large color baseline as one of their motivators. They utilized the entire upper RGB, establishing standard GC giant branches in the ( $\left.M_{I},(V-I)_{0}\right)$ plane. They demonstrated the substantial metallicity sensitivity of the $(V-I)_{0}$ color at a given $M_{I}$. This method now enjoys great popularity as the preferred technique for, e.g. deriving metallicities from photometric observations of the stellar populations in distant GCs and nearby resolved galaxies. Sarajedini (1994) further demonstrated that these standard RGBs could be used to determine both reddening and metallicity simultaneously, making the technique of even greater utility. Independently, a

number of investigators (e.g. Lee et al. 1993) have shown that the absolute I magnitude of the tip of the RGB (TRGB) is quite insensitive to metallicity and age over a wide range of both of these parameters, and can therefore be used as an accurate distance indicator, further enhancing the utility of $V, I$ photometry .

Given the distinct advantages of such a technique in many applications, the development of a similar technique using other filter combinations, including different photometric systems, is warranted. Geisler (1994) and Geisler and Sarajedini (1996) first introduced 
an analogous technique based on the $\left(C-T_{1}\right)$ color of the Washington photometric system. The Washington system (Canterna 1976a) is a four filter broadband system designed (Wallerstein and Helfer 1966) to provide an efficient yet accurate measurement of abundances and temperatures for $\mathrm{G}$ and $\mathrm{K}$ giants. In this regard, the system has proven useful for determining metallicities of individual giants in a variety of applications (cg. Geisler et al. 1991, hereafter GCM). GCM showed that the traditional two-color diagram technique utilizing Washington filters offered a powerful combination of efficiency and accuracy for determining metallicity in late-type giants over the full range of stellar abundances, though with decreased sensitivity for very cool, metal-poor stars, as is typical of similar techniques. The system has also proven useful for deriving the metallicity of distant GCs from their integrated color (cg. Harris and Canterna 1977, Geisler et al. 1996). In this latter application, the $\left(C-T_{1}\right)$ color has been successful because of its very high metallicity sensitivity compared to other indices such as $(B-V)$ and $(V-I)$ (Geisler et al. 1996). The $\left(C-T_{1}\right)$ index enjoys an even wider color baseline than $(V-I)$ while still falling within normal CCD response curves. Both filters are broad, with FWHM $>1000 \AA$. The $\mathrm{C}$ filter is similar to the Johnson $U$ filter, including the many spectral features in the blue-uv from $\sim 3500-4500 \AA$ that are metallicity sensitive in cool giants, but it is significantly broader and redder, making accurate photometry much more tractable, and reddening and atmospheric extinction less problematic. The $T_{1}$ filter is virtually identical to $R_{K C}$ (indeed, Geisler 1996 has recommended the substitution of the R(Kron-Cousins) filter for the $T_{1}$ filter, in particular because of its much higher efficiency) and offers a mostly continuum filter near the peak flux in cool giants and allows a wide color baseline in combination with $C$. Although the Washington system includes two other filters, $M$ and $T_{2}$, the desirability of deriving a technique, such as that of DCA, that uses only two filters to maximize telescope efficiency, is clear.

In view of the advantages outlined above, especially the efficiency and high metallicity 
sensitivity, and the fact that the $\left(C-T_{1}\right)$ index was already in use for the study of integrated GC colors, we have embarked on this investigation to establish standard giant branches in the Washington system using the $\left(C-T_{1}\right)$ color and $T_{1}$ magnitude. This work attempts to follow the high standards of photometric quality and analytical technique exemplified by the work of DCA. Throughout this paper, we compare our work to that of DCA because the latter has developed into a benchmark dataset used by many other investigators. Initial efforts were reported by Geisler (1994) and Geisler and Sarajedini (1996). Both of these papers demonstrated that such a technique has great potential for determining metal abundances in distant objects, with a metallicity sensitivity far exceeding that of $(V-I)$. However, these investigations were preliminary, with only a limited number of standard clusters, and photoelectric photometry for only a small number of stars per cluster.

This paper reports on the establishment of standard giant branches in the Washington system, based on CCD photometry for thousands of stars in each of a large number of well-studied Galactic GCs and old open clusters. The extensive photometry is described in Sect. 2. In Sect. 3, we present the standard giant branches. The metallicity calibration is detailed in Sect. 4, where we also compare the Washington standard giant branch technique with the existing Washington two-color diagram technique and the $(V-I)$ standard giant branch technique. In Sect. 5, we develop the technique to simultaneously determine reddening and metallicity. Sect. 6 describes how metallicity can also be determined from the RGB bump and slope, reddening from the color of the red edge of the blue HB and distance from the TRGB. We summarize our results in Sect. 7. 


\section{PHOTOMETRY}

\subsection{Sample Selection}

The choice of which clusters to select as 'standard' clusters is of paramount importance. Our primary sample selection criteria included: covering the full metallicity range of clusters in the Galaxy, with one cluster every $\sim 0.2$ dex in metallicity, using well-studied clusters with accurate metallicities, (small) distances and (low) reddenings - The standard clusters are listed in Table 1, which gives the NGC number, metallicity and reddening (from Zinn 1985 - hereafter Z85 - for the GCs except for NGC1851 whose metallicity has been revised, - see Sect. 4$),(m-M)_{V}$ and $[\mathrm{Fe} / \mathrm{H}]$ on two other metallicity scales. The distances will be discussed in Sect. 3. Note that this sample generally fulfills our criteria quite well. There are $10 \mathrm{GCs}$, ranging in metallicity (on the Z85 scale) from -2.15 to -0.3 . The reddenings are generally low, except for NGC5927. Note that 5 of these clusters - NGC7078 (M15), NGC6397, NGC6752, NGC1851 and NGC104 (47 Tuc) - are the same as used by DCA.

In order to cover even higher metallicities, we elected to use several well-studied old open clusters, NGC2682 (M67) and NGC6791. Although approximately solar metallicity GCs exist, they are very highly reddened and their distances and metallicities are only poorly determined. In contrast, these parameters are well known for our two selected open clusters. A potential problem in using open clusters is that age effects may become important if their ages are significantly less than those of the GCs. In this respect, NGC6791 is a perfect choice as it is one of the oldest open clusters known, with an age of $\sim 10 \mathrm{Gyr}$ (e.g. Tripicco et al. 1995) and thus quite comparable to those of the GCs in our sample. It also has a very high metallicity and is quite populous, allowing for accurate definition of the RGB. The case of NGC2682 is less fortuitous: it is only $\sim 4$ Gyrs old (e.g. Dinescu et al. 1995). Thus, age effects may start to play a role. However, it represents the oldest, 
nearest, most populated and best studied cluster of about solar metallicity. Possible age effects will be discussed in Sect. 4. The reddenings, metallicities and distances we have used for NGC2682 and NGC6791 represent means from a number of studies. Note that Twarog et al. (1997), in their recent compilation and homogenization of open cluster properties, give $E(B-V)=0.04,[\mathrm{Fe} / \mathrm{H}]=0.00 \pm 0.09$ and $(m-M)_{V}=9.68$ for NGC2682 and $0.15,0.15 \pm 0.16$ and 12.94 for the respective parameters of NGC6791. All of these values are very similar to ours except for the last one.

We then have 12 standard clusters, double the number used by DCA. Also, our standard clusters extend to metallicities 1 dex more metal-rich than those of DCA. At the same time, there is substantial overlap among the standard clusters of both samples, allowing for an accurate comparison of their respective advantages and disadvantages.

\subsection{Observations}

The observations were secured on a total of 14 nights from April, 1989 to December, 1996 using the CTIO 0.9m (7 nights), KPNO 4m (3 nights), KPNO 0.9m (3 nights) and CFHT (1 night). A variety of CCDs was used but generally a Tektronix $2048 \times 2048$ was the detector. The scale was typically $\sim 0.5^{\prime \prime} /$ pixel. Several different prescriptions for the Washington $\mathrm{C}$ and $T_{1}$ filters were used over this extended time period. The recommended prescription for $\mathrm{C}$ is that given in Geisler (1996): $3 \mathrm{~mm} \mathrm{BG3}+2 \mathrm{~mm} \mathrm{BG40.} \mathrm{For} T_{1}$, we used existing $T_{1}$ filters as well as a standard $R_{K C}$ filter. Geisler (1996) has shown that this filter is a more efficient substitute for $T_{1}$ and that $(C-R)$ accurately reproduces $\left(C-T_{1}\right)$ over the range $-0.2 \lesssim\left(C-T_{1}\right) \lesssim 3.3$. A few of the brightest, coolest giants in the most metal-rich and/or highly reddened clusters may exceed this color.

Each cluster was observed on at least 2 different nights (except for NGC2682). Each 
of these nights was photometric (except in the case of NGC7078 and NGC6791, where the single photometric night was used to calibrate the non-photometric night). Thus, we obtained two independent measurements for each cluster (with the noted exceptions).

Exposure times for the standard clusters varied from a few seconds to a few minutes. Care was taken to avoid saturating the brightest cluster giants, which are needed to define the TRGB. We typically obtained only a single exposure in each filter although occasionally several exposures were obtained and the median was reduced. The airmass was almost always $<1.5$ and the seeing generally ranged from $1-2^{\prime \prime}$. The cluster was usually centered on the CCD.

On each photometric night, a large number (typically 25-40) of standard stars from the lists of Geisler $(1990,1996)$ were also observed, some more than once. Care was taken to cover a wide color and airmass range for these standards in order to properly calibrate the program stars.

\subsection{Reductions}

Each frame was trimmed, bias-subtracted and flat-fielded (using twilight skyflats for C and either twilight skyflats or domeflats for $T_{1}$ ) using IRAF ${ }^{3}$ software.

The standard stars were measured with the aperture photometry routine APPHOT in IRAF. Since all of the standards lie in standard fields which include $\sim 10$ standard stars each, a mean value for the aperture correction (from an inner aperture $\approx$ FWHM to an

\footnotetext{
${ }^{3}$ IRAF is distributed by the National Optical Astronomy Observatories, which is operated by the Association of Universities for Research in Astronomy, Inc., under cooperative agreement with the National Science Foundation
} 
outer aperture $\approx 7^{\prime \prime}$ in radius) was applied. Transformation equations of the form:

$$
\begin{gathered}
c=C+a_{1}+a_{2} \times\left(C-T_{1}\right)+a_{3} \times X_{c} \\
t_{1}=T_{1}+b_{1}+b_{2} \times\left(C-T_{1}\right)+b_{3} \times X_{T_{1}}
\end{gathered}
$$

were used, where $c$ and $t_{1}$ refer to instrumental magnitudes (corrected to 1 s integration using a zero point of $25.0 \mathrm{mag}), \mathrm{C}, T_{1}$ and $\left(C-T_{1}\right)$ are standard values, and the appropriate air masses are given by X. We first solved for all three transformation coefficients simultaneously (using the PHOTCAL package in IRAF) for each night in a run and derived mean color terms for each filter in that run. We then substituted these mean color terms into the above equations and solved for the remaining two coefficients for each night simultaneously. The nightly rms errors from the transformation to the standard system ranged from 0.017 to 0.035 magnitudes in $\mathrm{C}$ and 0.010 to 0.020 magnitudes in $T_{1}$, with means of 0.023 and 0.016 magnitudes, indicating the nights were all of good to excellent photometric quality.

The program cluster data were reduced with the stand-alone version of the DAOPHOT II profile-fitting program (Stetson 1987). The standard FIND-PHOT-ALLSTAR procedure was generally performed 3 times on each frame, with typically some 20000 objects being measured in each cluster. A total of some 850,000 objects were photometered. After deriving the photometry for all detected objects in each filter, a cut was made on the basis of the profile diagnostics returned by DAOPHOT. Only objects with $\chi<2$, photometric error $<2 \sigma$ more than the mean error at a given magnitude, and $\mid$ Sharp $\mid<0.5$ were kept in each filter (typically discarding about $10 \%$ of the objects), and then the remaining objects in the $\mathrm{C}$ and $T_{1}$ lists were matched with a tolerance of 1 pixel, yielding instrumental colors and magnitudes. A quadratically varying PSF gave aperture corrections which were essentially constant with position except for data taken with the CTIO $0.9 \mathrm{~m}$ before 
1995 and data taken with the KPNO 4m before the new ADC was installed. In both of these instances, quadratically varying aperture corrections were required in addition to the quadratically varying psf. Mean aperture corrections were determined from 50-100 bright, unsaturated and uncrowded stars (after subtraction of all other photometered stars). The rms deviations about the mean were generally only 0.014 magnitude in $\mathrm{C}$ and 0.010 magnitude in $T_{1}$. These aperture corrections were then applied to all remaining objects and photometry on the standard Washington system was then obtained using the above transformation equations. Since no photometric CCD data was obtained for NGC2682, we used existing photoelectric data from Canterna (1976a) and GCM for 10 stars in common to transform the CCD data to the standard system. These stars covered a wide color range that included the full range of the RGB. Finally, the data of Friel and Geisler (1991) were used as one of the two observations for NGC5927.

\subsection{Final Photometry and Errors}

We then generally had two independent observations on the standard system for each cluster . In addition, most of the clusters also had photoelectric photometry available for a small number of giants from previous studies. The derivation of final colors and magnitudes was performed in the following manner. For the two clusters (NGC6791 and NGC7078) for which only a single photometric observation was available, these data were used to calibrate the non-photometric data, via a sample of several hundred bright stars with a wide color range to determine the zeropoint and color term in $T_{1}$ and $\left(C-T_{1}\right)$. The photometry was then averaged for all objects in all clusters which had two observations (this did not include all of the objects because of different field sizes and locations), leaving the photometry for single observations unchanged. For this procedure, we used Stetson's DAOMASTER routine, which provides the mean difference $(\Delta)$ and standard deviation $(\sigma)$ 
for the objects in common. This mean difference varied from 0.001 to 0.082 magnitudes for $\Delta(C)$, with a mean for all of the clusters of 0.038 magnitudes, while the corresponding $\sigma$ values ranged from 0.028 to 0.103 , with a mean of 0.059 magnitudes . Likewise, the $\Delta\left(T_{1}\right)$ values ranged from 0.002 to 0.042 , with a mean of 0.021 magnitudes, and the $\sigma\left(T_{1}\right)$ values ranged from 0.007 to 0.049 , with a mean of 0.026 magnitudes .

For the clusters which had existing photoelectric photometry (all except NGC5927), we then compared the CCD and photoelectric photometry for stars in common. Although photoelectric photometry of GC giants can suffer from crowding effects, the giants that had been observed were generally among the brightest in the cluster and were also selected for relative isolation, minimizing such effects. In addition, although crowding and photometric errors may be more severe than for the CCD profile-fitting photometry, these photoelectric observations are not subject to systematic errors such as flat-fielding and aperture corrections which can plague CCD data. The results of this comparison are given in Table 2. We list the mean difference (in the sense $C C D-P E$ ), $\sigma$ and the number of objects in common for both $\left(C-T_{1}\right)$ and $T_{1}$, as well as the source of the photoelectric photometry. Note that the comparison for NGC2682 is after transforming the CCD data to the photoelectric system, so that the mean differences are 0 by definition. Also note that two of the clusters (NGC5272 and NGC6791) had no $T_{1}$ photoelectric magnitudes available.

Figure 1 illustrates the resulting $\Delta\left(C-T_{1}\right)$ vs. $\left(C-T_{1}\right)_{P E}$ and $\Delta\left(T_{1}\right)$ vs. $\left(T_{1}\right)_{P E}$ diagrams for a representative cluster, NGC6397. In general, we find no significant trends of $\Delta$ with either color or magnitude. This holds even for very red stars $\left(\left(C-T_{1}\right)\right.$ 3.4) in NGC6791, among the reddest in our entire sample, which were observed using the $R_{K C}$ filter for $T_{1}$, giving confidence that such observations are not subject to large systematic errors (although we do not have photoelectric data available for even redder 
stars and thus cannot make such a statement for them). The mean differences (excluding NGC2682 and NGC104, which has only 1 star $)$ are: $\left\langle\Delta\left(C-T_{1}\right)\right\rangle=0.013 \pm 0.031(\sigma)$ and $\left\langle\Delta\left(T_{1}\right)\right\rangle=0.000 \pm 0.022$. This is quite satisfying agreement, indicating that our CCD photometry in general is very close to the photoelectric system. However, in the case of NGC4590, NGC6397 and NGC5272, the differences, especially for the colors, appear significant. For these 3 clusters, we offset the CCD photometry by the indicated differences to be on the photoelectric system.

The final Washington $T_{1}$ vs. $\left(C-T_{1}\right)$ color- magnitude diagrams (CMDs) for each cluster are shown in Figure 2. The photometry can be obtained from the first author upon request. From the above analysis, including nightly transformation errors, comparison of different CCD frames and the comparison of the CCD and photoelectric data, we conclude that this photometry is on the standard Washington system with zeropoint errors of $\sim 0.03$ magnitudes. These Washington CMDs show the same well-known features associated with these clusters from studies in other photometric systems: the main sequence and the turnoff, strong subgiant and RGBs, the variation of HB morphology with metallicity, the asympototic giant branch (AGB) and a smattering of field star contamination. The RGBs of the clusters in particular are very well defined, except for that of NGC5927, which suffers from severe field contamination as well as large and probably variable reddening. However, a reasonable RGB is still visible. (Note that, given the small size of the CCD in comparison to that of NGC2682, only a fraction of the cluster was covered and therefore we have supplemented the CCD data with the photoelectric data from Canterna (1976a) and GCM.) Of primary importance, it is clear that there is a very wide range in the color of the RGB between a low metallicity cluster like NGC7078 and a high metallicity cluster like NGC6791. 


\section{The Standard Giant Branches}

Because of uncertainties in reddenings and distances, it is best to derive the standard giant branches in the observational $\left(T_{1},\left(C-T_{1}\right)\right)$ plane before transforming them to the absolute magnitude, dereddened color $\left(M_{T_{1}},\left(C-T_{1}\right)_{0}\right)$ plane. Then, if the reddening or distance scale changes, they can be easily transformed again using the new values.

The analysis was generally confined to the brightest $\sim 5$ magnitudes of the RGB, which extends well below the level of the HB. The standard giant branches were manufactured by first excluding obvious HB, AGB and field stars. Then a third order polynomial was fit to the remaining points, further rejecting $>3 \sigma$ outliers. The cluster RGBs are very populous: even after this rejection, the fits involved an average of 361 stars in the 10 GCs. Note that the DCA fits typically used $<50$ stars. For the less-populated open clusters, only 14 giants were used in NGC2682 while 56 were available in NGC6791. The $T_{1}$ magnitude was employed as the independent variable (which can slightly bias the fits compared to a maximum likelihood solution). The rms of the fits in $\left(C-T_{1}\right)$ ranged from 0.028 (NGC4590, NGC6397 and NGC7078) to 0.117 (NGC5927), with a mean rms of 0.058 magnitudes. Note that this quantity does depend on the metallicity of the cluster, as the more metal-poor clusters have steeper RGBs. Although a third order polynomial was found to provide an excellent fit over virtually the entire RGB, some of the clusters (like NGC6752, NGC104 and NGC5927) exhibit extreme curvature at the TRGB, in some cases becoming fainter at the reddest colors, which was not well fit by the software. In order to account for this additional curvature, a hand-drawn fit was made which extended from the upper well-fit part of the polynomial through the reddest part of the RGB. In the case of NGC104, this hand-drawn curve was terminated at $\left(C-T_{1}\right) \sim 3.9$ although there are two stars lying much redder and fainter that could well belong to the cluster GB.

The fits are shown in Figure 3 and the coefficients given in Table 3. Only the fitted 
part of the RGB is shown. In each diagram, stars included in the fit are represented by filled boxes, excluded stars are open boxes, the large crosses are photoelectric observations and the solid curve is the third order polynomial fit (augmented by the hand-drawn curve for the TRGB in some instances). One can see again that the CCD data are generally in very good agreement with the photoelectric values. Note that the photoelectric data were not used in the fit, except in the case of NGC2682. The goodness of the fitted curves is also evident. The curves are of the form: $\left(C-T_{1}\right)=a+b\left(T_{1}-O\right)+c\left(T_{1}-O\right)^{2}+d\left(T_{1}-O\right)^{3}$, where $\mathrm{O}$ is the $T_{1}$ offset also given in the table.

To convert these fiducial GB curves to the $\left(M_{T_{1}},\left(C-T_{1}\right)_{0}\right)$ plane, a distance scale and cluster reddening must be adopted. We have utilized the Z85 reddenings for the GCs; those for the open clusters are based on a mean of the best available values. While the reddenings for these clusters are all relatively small and well known (except for that of NGC5927 and to a lesser extent that of NGC6352), the question of the appropriate distance scale remains controversial. This has become particularly true in the last year with the advent of the HIPPARCOS data. However, despite its intrinsic precision, application of the HIPPARCOS data to derive GC distances has produced several papers that are somewhat at odds (e.g. Reid 1997, Pont et al. 1998, Chaboyer et al. 1998) and the last word on the accuracy of this technique is not yet in. For this reason and to maintain consistency with DCA, we have adopted their distance scale, which in turn is based on the theoretical HB models of Lee et al. (1990). For these calculations, the luminosity of GC RR Lyrae variables follows the relation: $M_{V}(R R)=0.82+0.17[\mathrm{Fe} / \mathrm{H}]$. The variation of luminosity with abundance contained in this relation, which is appropriate for a helium abundance of $Y=0.23$, agrees to within the uncertainties with that derived from a variety of techniques, e.g. Baade-Wesselink analysis of field RR Lyraes (Carney et al. 1992) and the variation of HB magnitude with metallicity for M31 GCs (Fusi Pecci et al. 1996). However, as discussed in DCA, GCs with very red HBs and no RR Lyraes are problematical. Theoretical 
models (e.g. Lee et al. 1990) predict that the HB magnitudes of such clusters should be $\sim 0.1-0.2$ magnitudes brighter than hypothetical RR Lyraes. Again, to maintain consistency with DCA, we have adopted their value for this magnitude difference of 0.15 , i.e. $V(R R)$ is assumed to be 0.15 magnitudes fainter than the observed $V(H B)$. Similar values were obtained and used by Ajhar et al. (1996) and Fusi Pecci et al. (1996). Thus, to obtain a $(m-M)_{V}$ for $\mathrm{GCs}$, we use the $[\mathrm{Fe} / \mathrm{H}]$ value from $\mathrm{Z} 85$ and the Lee et al. relation to derive $M_{V}(R R)$. We then subtract this value from the observed $\mathrm{V}(\mathrm{HB})$ given in Armandroff (1989). For the exclusively red HB clusters (NGC104, NGC6352 and NGC5927) we finally add 0.15 . For the open clusters, we simply used the mean of the best existing $(m-M)_{V}$ determinations. Our $(m-M)_{V}$ values are given in Table 1 .

The standard giant branches are presented in Tables 4 and 5. Table 4 gives sample points at 0.1 magnitude intervals in $M_{T_{1}}$ from $M_{T_{1}}=+1$ to near the TRGB, while Table 5 gives additional points near the TRGB. Note that we have used $E\left(C-T_{1}\right)=1.97 E(B-V)$ and $A_{T_{1}}=2.62 E(B-V)$ (Geisler et al. 1996). Also, for $A_{V}=3.2 E(B-V)$, we have $M_{T_{1}}=T_{1}+0.58 E(B-V)-(m-M)_{V}$.

The standard giant branches for all 12 clusters are displayed together in Figure 4. The standard giant branches are generally well separated, with similar shapes, and ranked in order of metal abundance, with metallicity increasing from blue to red. It is also clear that the $M_{T_{1}}$ magnitude of the TRGB is roughly constant for the most metal-poor clusters and then increases with metallicity for the more metal-rich clusters. The color separation between the metallicity extremes is very large. This is graphically portrayed in Figure 5, which compares the $(V-I)$ standard giant branches from DCA with the $\left(C-T_{1}\right)$ standard giant branches for the same clusters. The Washington GBs are much more widely separated than the $(V-I)$ GBs. 


\section{Metallicity Determination}

\subsection{Metallicity Calibration}

The main goal of this study is to develop a technique which is a sensitive metallicity indicator and can be used to derive metallicity values in a wide range of applications. Following DCA, we will calibrate the $\left(C-T_{1}\right)_{0}$ color of the SGBs at a given $M_{T_{1}}$ as a function of metallicity. The selection of the fiducial $M_{T_{1}}$ value is of some importance. Clearly, as discussed by DCA and shown in Figure 4, the SGBs are more widely separated at brighter magnitudes. In addition, for application to distant stellar systems, one would like to have this fiducial magnitude as bright as possible, in order to obtain the most accurate photometry at this level. However, the more metal-rich SGBs do not reach as bright an $M_{T_{1}}$ as the more metal-poor clusters, and all of the SGBs are less well defined along the upper RGB than at fainter magnitudes. Therefore, the appropriate fiducial magnitude may be a compromise. Note that DCA first selected $M_{I}=-3$, about 1 magnitude below the TRGB, as their fiducial magnitude. However, in a subsequent paper (Armandroff et al. 1993), they developed the calibration for $M_{I}=-3.5$ due to the higher metallicity sensitivity, brighter magnitude and less confusion with AGB stars.

We have opted to derive metallicity calibrations for three different $M_{T_{1}}$ values: -2.5 , -2 and -1.5 . The middle value represents a point roughly 1 magnitude below the TRGB for the metal-poor clusters and is therefore comparable to DCA's value of $M_{I}=-3$. The brighter value will be more useful for distant, metal-poor systems while the fainter value is generally better defined (more stars available). Indeed, the most metal-rich GCs (NGC6352 and NGC5927) barely reach $M_{T_{1}}=-2$ and the two open clusters (NGC2682 and NGC6791) do not have stars at this magnitude. We have extrapolated the SGBs of these clusters to derive $\left(C-T_{1}\right)_{0}$ at $M_{T_{1}}=-2$ (and also slightly extrapolated that of NGC6791 to $M_{T_{1}}=-1.5$ ). There may still be a significant number of AGB stars present 
at this faintest magnitude but by $M_{T_{1}} \sim-2$ the AGB has generally blended with the RGB and should not have a significant effect on the mean $\left(C-T_{1}\right)$ color of the RGB. It is clear from Figure 4 that the Washington SGBs are still very well separated at even fainter magnitudes and that useful calibrations could be derived even below -1.5. Indeed, unlike the case of the $(V-I)$ SGBs, the $\left(C-T_{1}\right)$ SGBs still retain a very significant metallicity sensitivity even below the HB (see Fig. 5).

The choice of metallicity scale is also important. While the Z85 scale for Galactic GCs, which was used by DCA for their metallicity calibration, has been in vogue for many years and has generally held up well, several recent studies suggest a different scale may be more appropriate. In particular, Carretta and Gratton (1997, hereafter CG97) suggest, based on their high dispersion spectroscopic studies of a large number of GC giants, that the Z85 scale may be nonlinear with respect to the true $[\mathrm{Fe} / \mathrm{H}]$ scale. The extensive study by Rutledge et al. (1997) of Ca II triplet strengths supports the CG97 scale.

Again, we have opted to use three different metallicity scales: Z85, CG97 (as given in Rutledge et al. 1997) and our own "HDS" scale using the unweighted means of all high dispersion spectroscopic studies listed in Table 3 of Rutledge et al. but substituting the latest value of $[\mathrm{Fe} / \mathrm{H}](\mathrm{NGC7078})=-2.40$ from Sneden et al. (1997) for their earlier value of -2.30 (Sneden et al. 1991), which was based on many fewer stars. The metallicities for the standard clusters on each of these scales is given in Table 1. The Z85 metallicity for NGC1851 is -1.33 but a variety of studies (e.g. DCA, Armandroff and Zinn 1988, Armandroff and Da Costa 1991, Geisler et al. 1997) indicate that a more appropriate value is $\sim-1.15$ and that is the value we have adopted. Note that the latter two scales are genuine Fe abundance scales, i.e. they measure Fe abundances directly, while the Z85 scale is a "metallicity" scale involving many different techniques subject to different elemental abundances but generally Fe. The temperature of the RGB is mostly controlled by the 
primary electron donor elements $\mathrm{Mg}$ and $\mathrm{Si}$ as well as $\mathrm{Fe}$. Any differences among the relative abundances of these elements in different clusters could lead to significant temperature effects. This may in fact be the reason for the differences between the Z85 and CG97 scales. $\mathrm{Mg}$ and $\mathrm{Si}$ are generally found to be enhanced with respect to $\mathrm{Fe}$ in metal-poor GCs and not enhanced in solar abundance stars. However, there are some clusters (e.g. Pal 12 - Brown et al. 1997) that show different abundance patterns than normal for their metallicity. Such details should be born in mind when deriving a metallicity using the SGB and similar techniques.

Thus, we will derive 9 different calibrations: one for each combination of fiducial $M_{T_{1}}$ and metallicity scale, and the reader can choose whichever they feel is most appropriate or take an average of the metallicities derived from different calibrations. We also remind the reader that these calibrations depend on the reddening and choice of distance scale as well.

For each combination of $M_{T_{1}}$ and metallicity, we derived both linear and quadratic calibrations. The equations were of the form: $[\mathrm{Fe} / \mathrm{H}]=a+b\left(C-T_{1}\right)_{o}$, and $[\mathrm{Fe} / \mathrm{H}]$ $=a+b\left(C-T_{1}\right)_{o}+c\left(C-T_{1}\right)_{0}^{2}$. The linear equation was adopted unless the rms of the quadratic equation was significantly smaller. The coefficients, final number of clusters used and the rms values of the fits (in dex) are given in Table 6. All clusters available were used in all fits with equal weight except that NGC5927 was discarded from the $(-2, Z 85)$, $(-1.5, \mathrm{Z} 85),(-2, \mathrm{HDS})$ and $(-1.5, \mathrm{HDS})$ fits because of its discordant position. This is not unexpected given the wide RGB and substantial contamination and reddening of this disk cluster. The calibration curves are shown in Figure 6. The discarded NGC5927 points are indicated in parentheses.

From the table and figure, it is clear that some of the calibrations are much better defined than others. The lowest rms values at each $M_{T_{1}}$ are obtained for the Z85 scale, followed by the HDS scale. The CG97 calibrations for -2 and -1.5 are the poorest, with 
rms values of $\sim 0.12$ dex. All three $Z 85$ calibrations yield equally small rms values, but the $(-2, Z 85)$ calibration is particularly impressive as it includes all of the clusters except for NGC5927 and still gives an rms of only $\mathbf{0 . 0 4}$ dex (even including NGC5927 the rms is only $0.07 \mathrm{dex}$ ). This is our preferred calibration. Note that this is the one most analogous to that of DCA for which they obtained an rms of 0.07 dex using 8 clusters and a quadratic fit. In the figure associated with this calibration we also plot the five

clusters from DCA (shown as plus signs) which are common to both samples. Here we have added 1 to the $(V-I)_{0,-3}$ value from DCA (to facilitate comparison with the Washington values) and have used the Z85 metallicities for the DCA clusters, which in some cases are slightly different from the values they used. The much higher metallicity sensitivity of the Washington technique for the same clusters is striking.

This is then a very powerful but also simple technique for determining metallicities . After obtaining the photometry, the distance (on the Lee et al. 1990 scale) and reddening (both of which can be determined with the same photometry - see Sections 5 and 6) are used to place the data in the $\left(M_{T_{1}},\left(C-T_{1}\right)_{0}\right)$ plane. The $\left(C-T_{1}\right)_{0}$ value at the fiducial $M_{T_{1}}$ value (as determined from a fit) is then used in conjunction with the appropriate calibration to derive a metallicity. In practice, some iteration may be required, since the distance derivation may need an assumed metallicity. Alternatively, the observed RGB can be compared directly to the standard giant branches and a metallicity value interpolated.

\subsection{Comparison to Other Techniques}

\subsubsection{Comparison to the Washington Two-Color Diagram Technique}

The Washington system has long been used to determine the metallicity of $G$ and $\mathrm{K}$ giants. The traditional use of this system, most recently described in GCM, involves 
observations in 3 or 4 filters - C,M, $T_{1}$ and $T_{2}$ - where the metallicity is determined by the position of a giant in a two-color diagram plotting a color index mostly sensitive to metallicity (e.g. $\left.\left(C-T_{1}\right)\right)$ vs. an index mostly sensitive to temperature (e.g. $\left.T_{1}-T_{2}\right)$. It is important to see how well the new standard giant branch technique compares with the traditional technique for determining metallicities .

As discussed in GCM, one of the key measures of the suitability of a technique for determining metallicities is its metallicity sensitivity, S, defined (Trefzger 1981) as the change in the metallicity index for a given change in metallicity. For the two-color diagram Washington technique, $\mathrm{S}$ varies from a very low 0.04 (for the coolest, most metal-poor giants) to a very high 0.48 (for warmer, solar-abundance giants) and also depends on which combination of filters is used. For the new standard giant branch technique, at least for the linear calibrations, $\mathrm{S}$ is given by the inverse of the $\mathrm{b}$ coefficient in Table 6 . For these linear calibrations, $\mathrm{S}$ is a constant, independent of metallicity or temperature. For our preferred $(-2, Z 85)$ calibration, $S=0.79$. Thus, the new technique is some $\mathbf{1 . 6}$ to 20 times more metallicity sensitive than the old two-color diagram technique - In addition, the coolest, brightest giants fell outside the metallicity calibration of the two-color diagram technique and could not be used, despite their obvious advantage in terms of photometric precision. With the new technique, all stars along the RGB can be used.

Another important criterion of the utility of a metallicity determination technique is its sensitivity to photometric errors. For a given photometric error, the bigger its effect on the metallicity determination the less useful the technique. This criterion is also discussed in GCM for the two-color diagram technique. We use the same photometric error as they used, namely $\sigma\left(C-T_{1}\right)=0.025$, which is a typical number, and $\sigma\left(T_{1}\right)=0.02$. For the two-color diagram method, typical photometric errors lead to metallicity errors of from 
0.09 dex for intermediate temperature, metal-rich giants to 0.83 dex for cool, metal-poor giants, again also depending on the choice of color indices. For the standard giant branch technique and our preferred calibration, we derive a total metallicity error of only 0.034 dex. This is 2.7 to 25 times better than the two-color diagram method, which is not unexpected given the relative metallicity sensitivities. If we use a more appropriate error of 0.03 in both indices, the total metallicity error is still only 0.042 dex.

Thirdly, we also address the issue of how sensitive a metallicity index is to reddening , measured as the change in metallicity caused by a given change in the reddening. GCM found that an increase in the assumed reddening of $\Delta E(B-V)=+0.03$ led to an increase in the derived metallicity of from 0.02 to $0.60 \mathrm{dex}$, with the coolest, most metal-poor giants again showing the greatest sensitivity. For this same reddening increase, the metallicity derived from the standard giant branch method is decreased by 0.12 dex (again using our preferred calibration .) So in this case, for warm, solar abundance giants, the two-color diagram technique is actually less reddening sensitive than the new technique, while for metal-poor giants $([\mathrm{Fe} / \mathrm{H}]<-1)$ the new method is much less affected by reddening .

These three comparisons show that, under most circumstances, the new standard giant branch technique is a much better metallicity indicator than the two-color diagram method. For studies of approximately solar metallicity stars, say in old open clusters where the reddening is uncertain, the two-color diagram is still competitive, with a smaller reddening sensitivity but also smaller metallicity sensitivity and more sensitivity to photometric errors. Also, the two-color diagram method does not require knowledge of the distance. And, as discussed in 4.3, age effects using the new technique become important for objects younger than $\sim 5 \mathrm{Gyr}$, which includes most open clusters. However, in all other instances, especially for metallicities below $[\mathrm{Fe} / \mathrm{H}] \sim-1$, the new technique is far superior in all respects to the two-color diagram method. Clearly, 
the standard giant branch method also holds an important edge in observational efficiency in view of the need to only observe in two as opposed to three or four filters.

The study of very metal-poor objects will especially benefit from the use of the new technique. This is graphically illustrated by reexamining the metallicities of 5 metal-poor GCs studied by Geisler et al. (1992). In this study, they used the two-color diagram method to derive metallicities of a number of metal-poor Galactic GCs and found 5 of their sample - NGC2298, NGC4590, NGC4833, NGC5897, and NGC6101 - to have surprisingly low derived metallicities of $\sim-2.5$, some 0.4 to 0.8 dex more metal-poor than their Z85 metallicities, and more metal-poor than any other GCs known. As emphasized by Geisler et al. (1992), the reddenings of these clusters are generally relatively poorly known and the metallicity errors large. They recognized the limitations of their study and pointed to the need for further investigations, which subsequently did indeed generally confirm the Z85 metallicities (McWilliam et al. 1992, Minniti et al. 1993, Geisler et al. 1995). To illustrate the power of the new technique, we have derived Inetallicities for these clusters using the photometry of Geisler et al. (1992) and the preferred calibration of our standard giant branch technique. (Note that NGC4590 is one of our standard clusters but that the photometry is different). We find metallicities of $-1.71,-2.04,-1.97,-1.83$, and -1.86 for NGC2298, NGC4590, NGC4833, NGC5897 and NGC6101, respectively. These values compare very well with their Z85 values, with a mean difference $<[\mathrm{Fe} / \mathrm{H}]_{S G B}-[\mathrm{Fe} / \mathrm{H}]_{Z 85}>=-0.04 \pm 0.11(\sigma)$, compared to a mean difference of $-0.66 \pm 0.16$ using the two-color diagram method. The spuriously low metallicities derived from the two-color diagram technique are undoubtedly due to the lower metallicity sensitivity and higher reddening and photometric error sensitivity of this method, especially for low metallicity, cool giants. 


\subsubsection{Comparison to the $(V-I)$ Standard Giant Branch Technique}

We will also compare our technique to the prototype DCA $(V-I)$ standard giant branch technique using the same criteria (and preferred calibration) as above. The $(V-I)$ technique has been in vogue since DCA introduced it. Its popularity has even led to the selection of the corresponding F555W and F814W filters on the WFPC2 on HST as the standards for stellar population work with this important instrument.

The most direct comparison of the metallicity sensitivity of the two techniques is to compare the total difference in color index between the same GC standard giant branches at a similar fiducial magnitude. As noted above, there are 5 GCs in common to the two studies, and $M_{I}=-3$ is roughly comparable to $M_{T_{1}}=-2$. The total difference in $(V-I)_{0}$ between NGC104 and NGC7078 (the most metal-rich and metal-poor clusters in DCA, respectively) at this magnitude is 0.381 , while these same standard giant branches differ by 1.164 in $\left(C-T_{1}\right)_{0}$. Thus, the Washington technique is more than 3 times as metallicity sensitive as the $(V-I)$ technique. Note that the actual metallicity calibration derived by DCA between these clusters is not linear, as assumed here, but quadratic. Thus, the Washington technique will be even more sensitive at lower metallicities but $<3$ times as sensitive at higher metallicities. If one instead uses a fiducial magnitude that is 0.5 magnitude brighter, the Washington technique is only about 2.5 times as metallicity sensitive, still a substantial advantage.

To compare the relative photometric error sensitivities, we use the same photometric errors as above: 0.025 in $(V-I)$ and 0.02 magnitudes in I. Since the effect of magnitude errors is much less significant than that of color errors in this regard, one expects that the relative photometric error sensitivities will be similar to the metallicity sensitivities derived above. Indeed, one finds that the Washington technique is 2.9 times less sensitive to photometric errors as the $(V-I)$ technique at $M_{I}=-3$. In other words, for a 
given photometric accuracy, metal abundances can be determined 3 times more precisely with $\left(C-T_{1}\right)$ than $(V-I)$.

To compare the reddening sensitivities, we use $E(V-I) \sim 1.34 E(B-V)$ and $A_{I} \sim 1.55 E(B-V)$ for $R=A_{V} / E(B-V)=3.2$ (Dean et al. 1978). For an increase in the assumed reddening of $\Delta E(B-V)=+0.03$, the derived $(V-I)$ metallicity is decreased by about 0.19 dex, compared to 0.12 dex for the $\left(C-T_{1}\right)$ metallicity. So the Washington technique has only about half of the reddening sensitivity, in terms of its effect on metallicity, as the $(V-I)$ method.

Thus, the Washington technique enjoys a significant advantage over the corresponding $(V-I)$ technique for determining accurate metallicities. However, it is important to also compare their observing efficiencies. We determined the metallicity accuracies achieved in a given time for a given magnitude (namely 1 magnitude below the TRGB) with the two techniques. We investigated both a metal-poor $([\mathrm{Fe} / \mathrm{H}]=-2)$ giant and an intermediate metallicity $([\mathrm{Fe} / \mathrm{H}]=-1)$ giant, and used the appropriate colors based on the standard giant branches. We employed the count rates given in the latest KPNO Direct CCD Observing Manual for the KPNO 4m telescope, new ADC corrector and T2KB CCD. We assumed no moon and $1^{\prime \prime}$ seeing in all filters except for $1.2^{\prime \prime}$ in C. In a total integration time of 1 hour on an intermediate metallicity giant with $V=23.6$, a photometric error corresponding to a metallicity error of 0.10 dex was achieved in $(V-I)$ while a metallicity error of only $0.36 \mathrm{dex}$ was obtained in $\left(C-T_{1}\right)$. However, for the metal-poor giant, the roles were reversed, with $(V-I)$ achieving only a 0.23 dex metallicity error while $\left(C-T_{1}\right)$ yielded an error of 0.20 dex. Of course, this does not take into account aperture correction errors (which would tend to favor $\left(C-T_{1}\right)$ ), errors in transformation to the standard system (which would tend to favor $(V-I)$ ), etc. But in general it appears that $(V-I)$ is more efficient at obtaining precise metallicities for objects with $[\mathrm{Fe} / \mathrm{H}] \gtrsim$ 
-1.5 while $\left(C-T_{1}\right)$ wins for more metal-poor objects. Note that further improvements in the quantum efficiency of CCDs in the blue-uv will help to improve the C sensitivity , but similarly care must be taken to maintain maximum throughput in the optics in this spectral region.

We note that standard giant branches do exist in $V,(B-V)$ (Sarajedini and Layden 1997). They find that the NGC104 and NGC7078 standard giant branches are separated by 0.743 magnitudes in $(B-V)_{0}$ at $M_{V}=-2$, so the corresponding $V,(B-V)$ metallicity sensitivity is slightly $<2 / 3$ that of the Washington system. A comparison with their metallicity calibrations used in deriving the equivalent of the SRM method shows that, for their 6 calibrating clusters, they obtained rms values of from 0.05 to 0.08 dex, roughly comparable to our values.

\subsection{Age Effects}

The well known degeneracy between age and metallicity effects for stellar populations must be addressed. The standard clusters comprising our sample range in age from $\sim 4$ Gyr for NGC2682 to 10-15 Gyrs for the other clusters (depending on the distance scale adopted for the GCs and the selected isochrones). The two old open clusters were added to establish the metal-rich end of the metallicity calibration, with the hope that age effects would be small. How well was this hope born out? Clearly, given the very old age $(\sim 10$ Gyr) of NGC6791, age effects relative to those for the GCs should be minimal. And indeed a glance at Figure 6 verifies this. What about the much younger cluster NGC2682? Figure 6 shows that M67 does indeed lie at a slightly bluer color than expected for its metallicity in all calibrations in which it is involved. However, the effect is small, amounting to only about 0.1 dex or less. Such an effect could actually be due to our assumed metallicity being slightly too high. But it is consistent with the younger age causing a bluer RGB and 
leading to an underestimate of the metallicity .

Thus, empirically it appears that our metallicity calibrations may be safely applied to any cluster older than $\sim 4$ Gyr with only a small effect on the derived metallicity. An analysis based on Washington isochrones (Lejeune 1997) supports this. These isochrones include $U B V R I J H K$ as well as $C M T_{1} T_{2}$ and thus results from different color systems can be directly compared. An indication of the reliability of these isochrones is given by the fact that they yield a color difference in $\left(C-T_{1}\right)_{0} \quad$ (at $\left.M_{T_{1}}=-2\right)$ that is 3.16 times larger than the corresponding $(V-I)_{0}$ difference (at $\left.M_{I}=-3\right)$ between $[\mathrm{Fe} / \mathrm{H}]=-2.2$ and -0.6 (15 Gyr) isochrones, in excellent accord with the results found in the last section for the differences between the NGC104 and NGC7078 standard giant branches. The 8 and $15 \mathrm{Gyr},[\mathrm{Fe} / \mathrm{H}]=-1.6$ isochrones are separated by 0.093 magnitudes in $\left(C-T_{1}\right)_{0}$ at $M_{T_{1}}=-2$ and by 0.028 magnitudes in $(V-I)_{0}$ at $M_{I}=-3$. These color differences would lead to very similar metallicity "errors" of 0.12 and 0.11 dex, respectively, if gone unrecognized. Similarly, the differences between 8 and $15 \mathrm{Gyr},[\mathrm{Fe} / \mathrm{H}]=-0.6$ isochrones are 0.231 and 0.071 , leading again to very similar metallicity errors of 0.29 and 0.27 dex, respectively. As quoted in DCA, the Revised Yale Isochrones (Green et al. 1987) indicate that a $7 \mathrm{Gyr},[\mathrm{Fe} / \mathrm{H}]=-1.3$ isochrone is 0.05 magnitude bluer in $(V-I)_{0}$ at $M_{I}=-3$ than a 15 Gyr isochrone of the same metallicity, leading to a metallicity error of 0.2 dex, in very good agreement with the trend from the above isochrones. So the age sensitivity of the Washington standard giant branch technique appears to be very similar to, and perhaps slightly higher than, that of the $(V-I)$ method for old clusters, and both systems are more affected by age at higher than lower metallicities .

What about for even younger clusters? Clearly, by a certain age, the RGB will be moved sufficiently to the blue relative to an older cluster of the same metallicity that the effect on the derived metallicity will be significant. The Lejeune isochrones indicate 
that the age sensitivity becomes significant for clusters 5 Gyr and younger, especially for the Washington system. A similar value is obtained from the recent analysis of Bica et al. (1998) where they compared metallicities derived from the Washington standard giant branch technique with those available from spectroscopic studies for 5 Galactic open clusters and 6 LMC clusters whose ages ranged from 1-4 Gyrs (most were $\sim 2$ Gyr). For this sample, a clear trend was found for the Washington standard giant branch metallicities to underestimate the spectroscopic metallicity by an approximately constant amount, independent of age. An unweighted mean yielded a difference of $0.41 \pm 0.21$ dex. Thus, the standard giant branch technique derived here should only be applied to clusters older than $\sim 5$ Gyr.

We note in passing here that the Lejeune isochrones can also be used to test how well the Washington system works for deriving ages from main sequence photometry . VandenBerg et al. (1990) and Sarajedini and Demarque (1990) have shown that the color difference between the turnoff and the lower subgiant branch is a sensitive and powerful age indicator. For a $[\mathrm{Fe} / \mathrm{H}]=-0.6$ isochrone, this color difference is $\sim 0.27$ and 0.17 magnitudes in $(V-I)_{0}$ for a 10 and 20 Gyr population, while in $\left(C-T_{1}\right)_{0}$ the respective values are 0.71 and 0.47 magnitudes. Thus, the difference in these two values is some 2.5 times larger in $\left(C-T_{1}\right)_{0}$ than $(V-I)_{0}$, indicating that one could indeed obtain significantly greater age accuracy using the Washington system for a given photometric accuracy. These same age isochrones are separated by $\sim 0.13$ magnitudes in both color indices for $[\mathrm{Fe} / \mathrm{H}]=-2.2$ so for these lower metallicities the age sensitivity is the same. The Washington system thus holds great potential for deriving accurate ages as well as metallicities . 


\subsection{Intracluster Metallicity Dispersion}

As developed by DCA, one can use the dispersions in the fit of the standard giant branches to the data to derive upper limits to the intrinsic metallicity dispersion in each cluster . In order to investigate this quantity, we have used all cluster stars which fell between $M_{T_{1}}=-1.5$ and -2.5 , only excluding those stars that fell far away from the RGB. The standard devation, $\sigma\left(C-T_{1}\right)$, about the fitted standard giant branch was calculated and this value was converted into a metallicity dispersion using our preferred calibration . The results are displayed in Table 7, where we give the number of stars and the standard deviations in color and metallicity. Note that the two open clusters did not have sufficient stars in this part of the RGB to be useful. Also note that this is an upper limit to the intrinsic metallicity dispersion, as we have not taken photometric errors or contributions by AGB stars or field stars into account.

The results generally show very small upper limits to the intrinsic metallicity dispersion of our standard clusters, with a typical limit of $\sim 0.06$ dex. The limit for NGC5927 is especially large due to the presence of significant field star contamination as well as differential reddening. Our limits are generally similar to or lower than those derived for the same clusters by DCA, who found limits of 0.04 dex for NGC104, 0.07 for NGC1851, 0.06 for NGC6397, 0.05 for NGC6752, and 0.09 for NGC7078. Recall that our

observations were generally centered on these crowded clusters, whereas the DCA data were generally offset, leading to increased photometric error in the former with respect to the latter.

Such a procedure can be used to determine the metallicity dispersion in program objects. In this instance, the measured photometric error can be subtracted in quadrature from the observed scatter to derive a realistic estimate for any intrinsic dispersion. Indeed, given a sufficient sample in a system such as a dwarf spheroidal galaxy in which an intrinsic 
metallicity dispersion is expected, one could even derive the metallicity distribution of the giants using this technique, as was done by Geisler and Sarajedini (1996).

\section{Simultaneous Reddening and Metallicity Determination}

As noted in the Introduction, Sarajedini (1994) devised the simultaneous reddening and metallicity (SRM) technique to facilitate the determination of these quantities in an internally consistent manner. The SRM method exploits the fact that the shape and position of the RGB are dependent on metallicity and reddening. In addition to the calibration of these quantities using standard RGB sequences, the SRM method also requires knowledge of the $\mathrm{HB}$ magnitude, the color of the $\mathrm{RGB}$ at the level of the $\mathrm{HB}$, and the shape of the RGB.

The first step in establishing the SRM calibration is to estimate the value of $T_{1}(H B)$ for each cluster. This is a rather complicated endeavor because of the diversity of HB types among the clusters. For the clusters with RR Lyraes, we proceed as follows. Working in the $\left(T_{1}, C-T_{1}\right)$ plane, we fit a cubic polynomial to the HB stars that straddle the RR Lyrae instability strip. This is done using an iterative $2 \sigma$ rejection algorithm similar to that utilized for the RGB fits above. Based on the work of Sandage (1990), we have that the color at the blue edge of the RR Lyrae instability strip is $(B-V)_{0}=0.18$ and the color of the red edge is $(B-V)_{0}=0.40$. Converting these colors to $\left(C-T_{1}\right)_{0}$ with the transformations of Geisler (1996) gives a mean color of $\left(C-T_{1}\right)_{0}=0.45$ for the center of the instability strip. For those clusters with RR Lyraes, we read off $T_{1}(H B)$ from the polynomial fits at $\left(C-T_{1}\right)_{0}=0.45$. The rms of the fit added in quadrature with the uncertainty in the photometric zeropoint of $0.03 \mathrm{mag}$ is adopted as the error in $T_{1}(H B)$.

In the case of the two clusters with purely blue HB morphologies, NGC6397 and 
NGC6752, we use a different approach. First, we fit a polynomial to the blue HB stars in NGC5272. We then shift the HBs of NGC6397 and NGC6752 in $T_{1}$ until the mean star-by-star difference with the NGC5272 blue HB fit is minimized. The color shift is set by the difference in reddening between each cluster and NGC5272. Then, knowing $T_{1}(H B)$ for NGC5272 from the procedure described above and the shift required to match the blue HBs, we can infer the values of $T_{1}(H B)$ for NGC6397 and NGC6752. The error is estimated by adding in quadrature the uncertainty in the $T_{1}(H B)$ value of NGC5272 and the uncertainty in the shift.

There are 4 clusters with purely red HBs in our sample. We are omitting NGC2682 from the SRM calibration because its RGB is too sparsely populated. In the case of these clusters, we construct $T_{1}$ mag histogram distributions of the HB stars. Fitting a gaussian curve in the region of the peak in these histograms yields the value of $T_{1}(H B)$, while the error in $T_{1}(H B)$ is given by the dispersion in the fitted gaussian divided by the square root of the number of points used in the curve fit. The resulting error ends up being quite small (typically $<0.01 \mathrm{mag}$ ) because of the large number of points on the red $\mathrm{HB}$. The only significant error in the $T_{1}(H B)$ of the red $\mathrm{HB}$ clusters is that associated with the photometric zeropoint, which we estimate to be $\sim 0.03 \mathrm{mag}$. We note in passing that fitting the Gaussian in the region of the histogram peak minimizes the uncertainties in the value of $T_{1}(H B)$ introduced by the evolution of stars away (brightward) from the Zero Age Horizontal Branch.

Once we have settled on values of $T_{1}(H B)$ for each cluster, then we utilize the polynomial RGB fits to read off the $\left(C-T_{1}\right)_{g}$ value, which is converted to $\left(C-T_{1}\right)_{0, g}$ by applying our adopted reddenings. We can also construct the magnitude difference between the HB and RGB at $\left(C-T_{1}\right)_{0}=2.4$ (i.e. $\left.\Delta T_{2.4}\right)$. Both of these quantities, $\left(C-T_{1}\right)_{g}$ and $\Delta T_{2.4}$ vary with metal abundance, and are listed in Table 8 along with the other measured 
parameters. Performing a weighted least squares fit gives us the two relations that are central to the SRM method,

$$
\begin{gathered}
{[\mathrm{Fe} / \mathrm{H}]=a_{1}+b_{1} \times\left(C-T_{1}\right)_{0, g}+c_{1} \times\left(C-T_{1}\right)_{0, g}^{2}+d_{1} \times\left(C-T_{1}\right)_{0, g}^{3}} \\
{[\mathrm{Fe} / \mathrm{H}]=a_{2}+b_{2} \times \Delta T_{2.4}+c_{2} \times \Delta T_{2.4}^{2}+d_{2} \times \Delta T_{2.4}^{3} .}
\end{gathered}
$$

Table 9 gives the values of these coefficients for the various metallicity scales considered herein, while Fig. 7 illustrates the relations. The metallicity errors are taken from Zinn \& West (1984) for the Z85 abundances and Rutledge et al. (1997) for the CG97 and HDS abundances.

The reader is referred to the work of Sarajedini (1994) for a detailed description of how to apply the SRM method. In summary, one needs a polynomial or fiducial representation of the cluster RGB sequence as well as estimates for the observed values of $T_{1}(H B)$ and $\left(C-T_{1}\right)_{g}$. Then, an iterative procedure can be set up that utilizes these observed properties in conjunction with the two equations above to provide estimates of the cluster reddening and metallicity. In addition, Monte Carlo simulations are used to determine the uncertainty in these quantities. Given a well-determined RGB sequence with errors of $0.03 \mathrm{mag}$ in $T_{1}(H B)$ and $0.03 \mathrm{mag}$ in $\left(C-T_{1}\right)_{g}$, we expect metallicity errors of $0.15 \operatorname{dex}$ in $[\mathrm{Fe} / \mathrm{H}]$ and reddening errors of $0.05 \mathrm{mag}$ in $E\left(C-T_{1}\right)(0.025 \mathrm{mag}$ in $\mathrm{E}(\mathrm{B}-\mathrm{V}))$. As for the effects of cluster age, Sarajedini \& Layden (1997) and Mighell et al. (1998) have shown that the SRM method is insensitive to age for clusters $\sim 4$ Gyr and older. 


\section{Additional Metallicity, Reddening and Distance Determination Techniques}

\subsection{Metallicity Determination from the Slope of the Red Giant Branch}

The work of Hartwick (1968) illustrated the suitability of the RGB slope as a metallicity indicator. In the present work, we define the RGB slope $\left(S_{-2}\right)$ as

$$
S_{-2}=\frac{-2.0}{\left[\left(C-T_{1}\right)_{g}-\left(C-T_{1}\right)_{-2}\right]},
$$

where $\left(C-T_{1}\right)_{-2}$ is the color of the RGB at 2 magnitudes above the HB. This quantity is well correlated with metal abundance; as such, we can construct the following relation -

$$
[\mathrm{Fe} / \mathrm{H}]=a+b \times S_{-2}+c \times S_{-2}^{2}
$$

for the three metallicity scales. Table 10 lists the values of these coefficients while Fig. 8 illustrates the relations. The RGB slope method of metallicity determination is especially useful since it does not require knowledge of the reddening or the distance. Furthermore, Mighell et al. (1998) have shown that, in the $B-V$ passbands, it is insensitive to age for clusters older than $\sim 4 \mathrm{Gyr}$. If the values of $\left(C-T_{1}\right)_{g}$ and $\left(C-T_{1}\right)_{-2}$ can be determined with an error of $\pm 0.03 \mathrm{mag}$, then the resultant error in $[\mathrm{Fe} / \mathrm{H}]$ is approximately $0.08 \mathrm{dex}$.

\subsection{Metallicity Determination from the Magnitude of the Red Giant Branch Bump}

As a star evolves up the first ascent RGB, it reaches a point where its evolution pauses or reverses course for a short time, after which it resumes its brightward movement in the H-R Diagram (Thomas 1967; Iben 1968). This phenomenon leads to a clumping of stars along the RGB. When a luminosity function (LF) is constructed, this clump manifests itself as a bump in the $L F$, hence the name 'RGB bump.' 
From theoretical considerations, the luminosity of the RGB bump is dependent on age and abundance (Iben 1968, Fusi Pecci et al. 1990). However, since the absolute zeropoint of the theoretical RGB bump luminosity is uncertain, it is more useful to measure the RGB bump magnitude relative to that of the HB (i.e. $\Delta T_{1}(B u m p-H B)$ ). In these terms, as a cluster's age and/or metallicity increases, $\Delta T_{1}(B u m p-H B)$ also increases. For clusters that are older than $\sim 10 \mathrm{Gyr}$, the effect of age on the absolute magnitude is negligible (Alves \& Sarajedini 1998); as a result, we can utilize the magnitude difference between the RGB bump and the HB as a metallicity indicator as proposed by Fusi Pecci et al. (1990) and parameterized by Sarajedini \& Forrester (1995).

To isolate the location of the RGB bump, we proceed in the same manner as Sarajedini \& Norris (1994). First, we construct a cumulative LF of the RGB stars. As illustrated in their Fig. 15, the RGB bump clearly stands out in such LFs. After fitting a 'continuum' to the LF around the region of the bump, we subtract this off resulting in a flattened RGB LF. The maximum point in this flattened LF represents the faintest extent of the RGB bump, while the zero crossing immediately brightward represents the onset of the bump as one proceeds fainter from the RGB tip. We therefore adopt the magnitude midway between these two points as the value of $T_{1}(B u m p)$, which is then coupled with $T_{1}(H B)$ to produce $\Delta T_{1}\left(\right.$ Bump - HB). The error in $T_{1}(B u m p)$ is half the distance between the maximum and the zero-crossing multiplied by 0.68 to simulate a $1-\sigma$ uncertainty.

As in the case of the RGB slope, we parameterize the variation of the RGB bump with metallicity using the following relations.

$$
[F e / H]=a+b \times \Delta T_{1}+c \times \Delta T_{1}^{2}
$$

where $\Delta T_{1}$ is the difference in magnitude between the bump and the HB; the fitted coefficients are listed in Table 11. Figure 9 shows the fitted points and the resulting relations. If the value of $\Delta T_{1}$ can be measured to \pm 0.1 magnitude, then the metallicity 
determined from the RGB Bump will have an uncertainty of $\sim 0.1$ dex.

Our CMDs are sufficiently populous that, at least in several instances, we see evidence of a similar LF enhancement on the AGB $\sim 1$ magnitude above the HB. The best cases are NGC104, NGC5272 and NGC7078. We believe that this feature is the AGB bump, due to a phenomenon similar to that which produces the RGB bump. Such a feature has

recently been identified in populous LMC field star CMDs (Gallart 1998) and may well be responsible for the 'VRC' suggested by Zaritsky \& Lin (1997) as being due to a possible foreground galaxy.

\subsection{Reddening Determination from the Red Edge of the Blue Horizontal Branch}

The temperature limits of the RR Lyrae instability strip are well-defined, both theoretically and observationally (see Sandage 1990). Previous studies have exploited this fact to derive the reddening of a cluster, given a large sample of RR Lyraes and giants along the BHB: the observed color limit between the variables and non-variables is compared to the intrinsic color limit, directly yielding the reddening. We have undertaken a similar analysis, using the six standard clusters (NGC1851, NGC4590, NGC5272, NGC6362, NGC6752 and NGC7078) which have both RR Lyraes and BHB stars. NGC6752 in fact does not have RR Lyraes but does appear to have BHB stars that lie very near the edge of the instability strip. On the other hand, the reddest BHB stars in NGC6397 fall substantially blueward of the instability strip.

It is clear from Figure 2 that the BHB stars in these clusters generally lie in a fairly tight sequence, that the RR Lyraes fall in a more scattered distribution from fainter, redder colors to brighter, bluer colors, and that the color limit between these two types of stars is 
reasonably well defined. We derived this limit in $\left(C-T_{1}\right)$ and converted it to $\left(C-T_{1}\right)_{0}$ using the cluster reddenings. The six clusters have $\left\langle\left(C-T_{1}\right)_{o}\right\rangle=0.17$, with a $\sigma$ of only 0.03 . Sandage $(1990)$ gives this limit as $(B-V)_{0}=0.18$ which is $\left(C-T_{1}\right)_{0}=0.22$ using the conversion of Geisler (1996). Giving some weight to this determination, we adopt $\left(C-T_{1}\right)_{0}=0.18 \pm 0.04$ for the intrinsic color of the red edge of the BHB. We can then use this value to derive the reddening to a program object which has a sufficient number of BHB and RR Lyrae stars, with an estimated error of $\sigma(E(B-V))=0.025$. This technique can be used to supplement the reddening derived from the SRM method described above.

\subsection{Distance Determination from the $T_{1}$ Magnitude of the Tip of the Red Giant Branch}

The I magnitude of the TRGB has become an increasingly popular standard candle in recent years. The work of Lee et al. (1993) and others has shown that this is indeed a very useful distance indicator. We have investigated the analogous use of the $T_{1}$ magnitude of the TRGB as a distance indicator.

A glance at Figure 4 suggests that $M_{T_{1} \text { RGB }}$ has at most a very small dependence on metallicity for metal-poor clusters $([\mathrm{Fe} / \mathrm{H}] \lesssim-1.15)$. We have determined this value for the 6 standard clusters falling in this regime. A mean $M_{T_{1}{ }_{T R G B}}=-3.22 \pm 0.11(\sigma)$ is obtained. The small $\mathrm{rms}$ indicates that this should indeed be a useful distance indicator for such objects. Clearly, though, at higher metallicities $M_{T_{1_{T R G B}}}$ increases rapidly with increasing metallicity and is not useful. Indeed, such behavior is also seen for $M_{I_{T R G B}}$ for $[\mathrm{Fe} / \mathrm{H}] \gtrsim-0.75$.

We can use the Bertelli et al. (1994) isochrones to investigate theoretical predictions

concerning how $M_{T_{1} \text { RGB }}$ depends on age and metallicity. Geisler (1996) has shown that 
the $T_{1}$ and $R_{K C}$ magnitude scales are virtually identical, with an almost negligible zeropoint (0.003) and color term (0.017) relating them. Therefore, we can simply use the $M_{R_{K C}}$ magnitudes generated by Bertelli et al. and compare them directly to our $M_{T_{1}}$ results. The results are shown in Figure 10 where $M_{T_{1_{T R G B}}}$ is plotted vs. $[\mathrm{Fe} / \mathrm{H}]$. The plus signs are from the Bertelli et al. models, where we have used an age of 12 Gyrs except for

the point at solar metallicity $(4 \mathrm{Gyr})$ and $[\mathrm{Fe} / \mathrm{H}]=+0.4(10 \mathrm{Gyr})$ to overlap with the ages of our standard open clusters at these metallicities. The squares are from our standard giant branches. The agreement between the models and observations is excellent. Both sets of data indicate that $M_{T_{\mathrm{l}_{T R G B}}}$ is very sensitive to metallicity for $[\mathrm{Fe} / \mathrm{H}] \gtrsim-1.2$ but that for more metal-poor clusters there is only a slight or possibly negligible metallicity dependence. Therefore, for such metal-poor objects, $M_{T_{1} \text { TRGB }}$ can indeed be used as an accurate distance indicator, with a value of -3.22 and an error of $\lesssim 0.15$ magnitudes, for ages from $\sim 3-20 \mathrm{Gyr}$.

\section{Summary}

We have obtained CCD photometry in the Washington system for a very large sample of stars in each of 10 Galactic GCs and 2 open clusters. The photometry is on the standard system to within $\lesssim 0.03$ magnitudes. We have fit third-order polynomials to each cluster to derive the standard giant branch. These standard giant branches are converted to the (absolute magnitude, dereddened color) plane by assuming the Lee et al. (1990) distance scale and reddenings from Z85. We then derive metallicity calibrations for the $\left(C-T_{1}\right)_{0}$ color at three different fiducial $M_{T_{1}}$ values, and for three metallicity scales. Our preferred calibration is for $M_{T_{1}}=-2$, about a magnitude fainter than the TRGB of metal-poor clusters, using the Z85 metallicity scale. This calibration is very analogous to that derived by DCA for VI photometry. We find that the Washington system enjoys 
three times the metallicity sensitivity of the VI technique. It is also less sensitive to reddening. The Washington standard giant branch metallicity technique is also superior to the Washington two-color diagram technique in virtually all respects. The standard giant branch technique is immune to age effects for objects older than $\sim 5$ Gyr. We derive upper limits of typically 0.06 dex for any intrinsic metallicity dispersion in the standard clusters .

We also use the standard giant branches to derive a method analogous to that of Sarajedini (1994) for determining both the metallicity and reddening simultaneously. The magnitude difference between the HB and the RGB bump, and the slope of the RGB, are also found to be sensitive metallicity indicators. In addition, reddening can be determined from the color of the red edge of the blue HB. Finally, the $T_{1}$ magnitude of the TRGB is an accurate distance indicator for objects more metal-poor than $[\mathrm{Fe} / \mathrm{H}] \sim-1.2$ and older than 3 Gyr. An analysis of available isochrones indicates that the Washington system also holds great potential for deriving accurate ages as well as metallicities .

Gary Da Costa and Taft Armandroff provided much of the inspiration for this work from their original study in the V,I system (DCA). We thank Hugh Harris for a critical reading of a preliminary version of this manuscript. The authors would like to thank Juan Clariá and Cristina Torres for providing important photoelectric data in advance of publication. Juan Clariá and Andrés E. Piatti also very kindly obtained one of the CCD fields for us. Pat Durrell allowed us to obtain another field during telescope time set aside for his thesis. Peter Stetson, as always, has been very generous in allowing the use of his own very useful programs. Jim Roberts and Eva Grebel are acknowledged for their initial work on another project which helped to launch the present one. The cluster photometry can be obtained from the first author upon request. This research is supported in part by NASA through grant No. GO-07265.01-96A (to DG) and HF-01077.01-94A (to AS) from 
the Space Telescope Science Institute, which is operated by the Association of Universities for Research in Astronomy, Inc., under NASA contract NAS5-26555. This research is supported in full by E. Geisler through her constant love and encouragement. 


\section{REFERENCES}

Ajhar, E.A., Grillmair, C.J., Lauer, T.R., Baum, W.A., Faber, S.M., Holtzman, J.A., Lynds, C.R., \& O’Neil, E.J. 1996, AJ, 111, 1110

Alves, D., \& Sarajedini, A. 1998, in preparation

Armandroff, T.E. 1989, AJ, 97, 375

Armandroff, T.E., \& Da Costa, G.S. 1991, AJ, 101, 1329

Armandroff, T.E., Da Costa, G.S., Caldwell, N., \& Seitzer, P. 1993, AJ, 106, 986

Armandroff, T.E., \& Zinn, R. 1988, AJ, 96, 92

Bertelli, G., Bressan, A., Chiosi, C., Fagotto, F., \& Nasi, E. 1994, A\&AS, 106, 275

Bica, E., Geisler, D., Dottori, H., Clariá, J.J., Piatti, A.E. \& Santos, J.F.C. Jr. 1998, AJ, in press

Brown, J.A., Wallerstein, G., \& Zucker, D. 1997, AJ, 114, 180

Canterna, R. 1976a, AJ, 81, 228

Canterna, R. 1976b, PhD. thesis, University of Washington

Canterna, R., Geisler, D., Harris, H.C., Olszewski, E., \& Schommer, R. 1986, AJ, 92, 79

Carney, B.W., Storm, J., \& Jones, R.V. 1992, ApJ, 386, 663

Carretta, E., \& Gratton, R.G. 1997, A\&AS, 121, 95 (CG97)

Chaboyer, B. , Demarque, P., Kernan, P. J., \& Krauss, L. M. 1998, ApJ, 494, 96

Clariá, J.J., \& Torres, M.C. 1998, in preparation

Da Costa, G.S., \& Armandroff, T.E. 1990, AJ, 100, 162 (DCA)

Dean, J.F., Warren, P.R., \& Cousins, A.W.J. 1978, MNRAS, 183, 569

Dinescu, D.I., Demarque, P., Guenther, D.B., \& Pinsonneault, M.H. 1995, AJ, 109, 2090 
Friel, E.D., \& Geisler, D. 1991, AJ, 101, 1338

Frogel, J.A., Cohen, J.G., \& Persson, S.E. 1983, ApJ, 275, 773

Fusi Pecci, F., Buonanno, R., Cacciari, C., Corsi, C.E., Djorgovski, S.G., Federici, L., Ferraro, F.R., Parmeggiani, G., \& Rich, R.M. 1996, AJ, 112, 1461

Fusi Pecci, F., Ferraro, F.R., Crocker, D.A., Rood, R.T., \& Buonanno, R. 1990, A\&A, 238, 95

Gallart, C. 1998, ApJ, 495, 43

Geisler, D. 1986a, PASP, 98, 762

Geisler, D. 1986b, PASP, 98, 847

Geisler, D. 1990, PASP, 102, 344

Geisler, D. 1994, in The Local Group: Comparative and Global Propeties, ESO Conf. Proc.

No. 51, ed. A. Layden, R.C. Smith \& J. Storm (Garching:ESO), 141

Geisler, D. 1996, AJ, 111, 480

Geisler, D., Clariá, J.J., \& Minniti, D. 1991, AJ, 102, 1836 (GCM)

Geisler, D., Clariá, J.J., \& Minniti, D. 1997, AJ, 109, 799

Geisler, D., Lee, M.G., \& Kim, E. 1996, AJ, 111, 1529

Geisler, D., Minniti, D., \& Clariá, J.J. 1992, AJ, 104, 627

Geisler, D., Piatti, A.E., Clariá, J.J., \& Minniti, D. 1995, AJ, 109, 605

Geisler, D. \& Sarajedini, A. 1996, in Formation of the Galactic Halo...Inside and Out, ASP

Conf. Ser., no.92, ed. H. Morrison \& A. Sarajedini (San Francisco:ASP), 524

Green, E.M., Demarque, P., \& King, C.R. 1987, The Revised Yale Isochrones and Luminosity Functions (Yale University Observatory, New Haven)

Harris, H.C., \& Canterna, R. 1977, AJ, 82, 798 
Hartwick, F.D.A. 1968, ApJ, 154, 475

Iben I. Jr. 1968, Nature, 220, 143

Lee., M.G., Freedman, W.L., \& Madore, B.F. 1993, ApJ, 417, 553

Lee, Y.-W., Demarque, P., \& Zinn, R. 1990, ApJ, 350, 155

Lejeune, T. 1997, Ph.D. thesis, Universite Louis Pasteur, Strasbourg

McWilliam, A., Geisler, D., \& Rich, R.M. 1992, PASP, 104, 1193

Mighell, K. J., Sarajedini, A., \& French, R. S. 1998, in preparation

Minniti, D., \& Clariá, J.J. 1989, ApJ, 341, 186

Minniti, D., Geisler, D., Peterson, R.C., \& Clariá, J.J. 1993, ApJ, 413, 548

Pont, F., Mayor, M., Turon, C., \& VandenBerg, D. 1998, A\&Ap, 329, 87

Reid, I.N. 1997, AJ, 114, 161

Rood, R.T. 1978, in Globular Clusters, NATO Advanced Study Institute

Rutledge, G.A., Hesser, J.E., \& Stetson, P.B. 1997, PASP, 109, 907.

Sandage, A.R. 1990, ApJ, 350, 603

Sandage, A.R., \& Smith, L.L. 1966, ApJ, 144, 886

Sarajedini, A. 1994, AJ, 107, 618

Sarajedini, A., \& Demarque, P. 1990, ApJ, 365, 219

Sarajedini, A., \& Forrester, W. L. 1995, AJ, 109, 1112

Sarajedini, A., \& Layden, A. C. 1997, AJ, 113, 264

Sarajedini, A., \& Norris, J.E. 1994, ApJS, 93, 161

Searle, L., \& Zinn, R. 1978, ApJ, 225, 357

Sneden, C., Kraft, R.P., Prosser, C.F., \&Langer, G.E. 1991, AJ, 102, 2001 
Sneden, C., Kraft, R.P., Shetrone, M.D., Smith, G.H., Langer, G.E., \& Prosser, C.F. 1997, $\mathrm{AJ}, 114,1964$

Stetson, P.B. 1987, PASP, 99, 191

Thomas, H. -C. 1967, Z. Ap, 67, 420

Trefzger, C.F. 1981, in Astrophysical Parameters for Globular Clusters, IAU Coll. No. 68, ed. A.G.D. Philip and D.S. Hayes (Davis, Schenectady), 33

Tripicco, M.J., Bell, R.A., Dorman, B., \& Hufnagel, B. 1995, AJ, 109, 1697

Twarog, B.A., Ashman, K.M., \& Anthony-Twarog, B.J. 1997, AJ, 114, 2556

VandenBerg, D.A., Bolte, M., \& Stetson, P.B. 1990, AJ, 100, 445

Wallerstein, G., \& Helfer, H.L. 1966, AJ, 71, 350

Zaritsky, D., \& Lin, D.N.C. 1997, AJ, 114, 2545

Zinn, R. 1985, ApJ, 293, 424 (Z85) 


\section{Figure Captions}

Fig. 1.- a. The difference between the $\left(C-T_{1}\right)$ value derived from CCD and photoelectric photometry, as a function of the photoelectric value, for giants in NGC6397 in common. b. As for a). except for the $T_{1}$ magnitude. 
Fig. 2.- $T_{1}$ vs. $\left(C-T_{1}\right)$ color-magnitude diagram for the standard clusters . a. NGC104 b. NGC1851 c. NGC2682 NB - in this diagram, CCD observations are denoted by squares and photoelectric data by crosses. d. NGC4590 e. NGC5272 f. NGC5927 g. NGC6352 h. NGC6362 i. NGC6397 j. NGC6752 k. NGC6791 1. NGC7078

Fig. 3. $-T_{1}$ vs. $\left(C-T_{1}\right)$ CMD for the standard clusters showing the standard giant branches. Filled squares are observations used in the fit, open squares are unused or rejected stars. Large crosses indicate photoelectric observations. The solid curve is the standard giant branch fit. a. NGC104 b. NGC1851 c. NGC2682 d. NGC4590 e. NGC5272 f. NGC5927 g. NGC6352 h. NGC6362 i. NGC6397 j. NGC6752 k. NGC6791 l. NGC7078

Fig. 4.- The Washington standard giant branches in the $\left(M_{T_{1}},\left(C-T_{1}\right)_{0}\right)$ plane. At $M_{T_{1}}$ $=-1.5$, the standard giant branches are (from left to right): NGC7078, NGC4590, NGC6397, NGC5272, NGC6752, NGC1851, NGC6362, NGC104, NGC5927, NGC6352, NGC2682 and NGC6791.

Fig. 5.-- A comparison of the same VI and Washington standard giant branches. The VI data are taken from DCA and plot $M_{I}$ vs. $(V-I)_{0}$. The Washington data plots $M_{T_{1}}$ vs. $\left(C-T_{1}\right)_{0}$. The clusters are (from left to right): NGC7078, NGC6397, NGC6752, NGC1851 and NGC104. The Washington standard giant branches are much more widely separated than the VI RGBs.

Fig. 6.- Metallicity calibrations for various combinations of fiducial magnitude and metallicity scale. a. $\left(M_{T_{1}}=-2.5, \mathrm{Z} 85\right)$ b. $(-2.5, \mathrm{CG} 97)$ c. $(-2.5, \mathrm{HDS}) \mathrm{d} .(-2, \mathrm{Z} 85)$. This is our preferred calibration. The discarded point (NGC5927) is shown in parantheses. The plus signs show the DCA standard giant branches, using $M_{I}=-3$ and adding 1 to $(V-I)_{0}$ e. $(-2$, CG97 $)$ f. $(-2$, HDS $)$ g. $(-1.5$, Z85 $)$ h. $(-1.5$, CG97) i. $(-1.5$, HDS $)$ 
Fig. 7.- Calibration of the SRM method for deriving reddening and metallicity simultaneously. $[\mathrm{Fe} / \mathrm{H}]$ (for three different metallicity scales) is shown as a function of the CMD parameters $\left(C-T_{1}\right)_{0,9}$ and $\Delta T_{2.4}$ for the standard clusters.

Fig. 8. - Calibration of the RGB slope for deriving metallicity . [Fe/H] (for three different metallicity scales) is shown as a function of the RGB slope.

Fig. 9. - Calibration of the RGB bump for deriving metallicity . $[\mathrm{Fe} / \mathrm{H}]$ (for three different metallicity scales) is shown as a function of $\Delta T_{1}($ Bump-HB).

Fig. 10.- $-M_{T_{1}}$ at the RGB tip as a function of metallicity. The squares are the standard giant branches; the plus signs are from the model isochrones of Bertelli et al. (1994). For metallicities $\lesssim-1.2, M_{T_{1_{T R G B}}}$ is nearly constant and can be used as a distance indicator. 



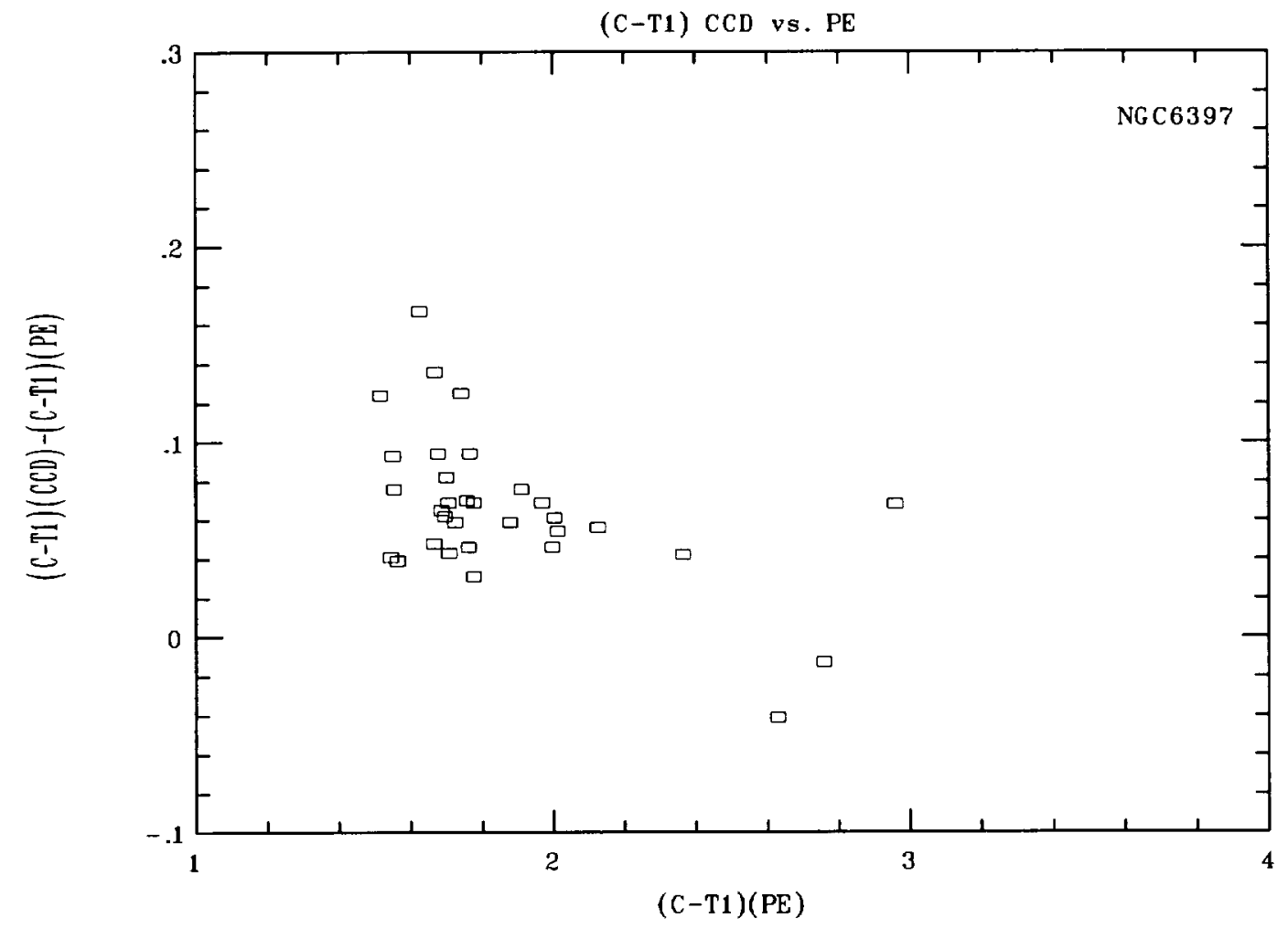

Fig.la 


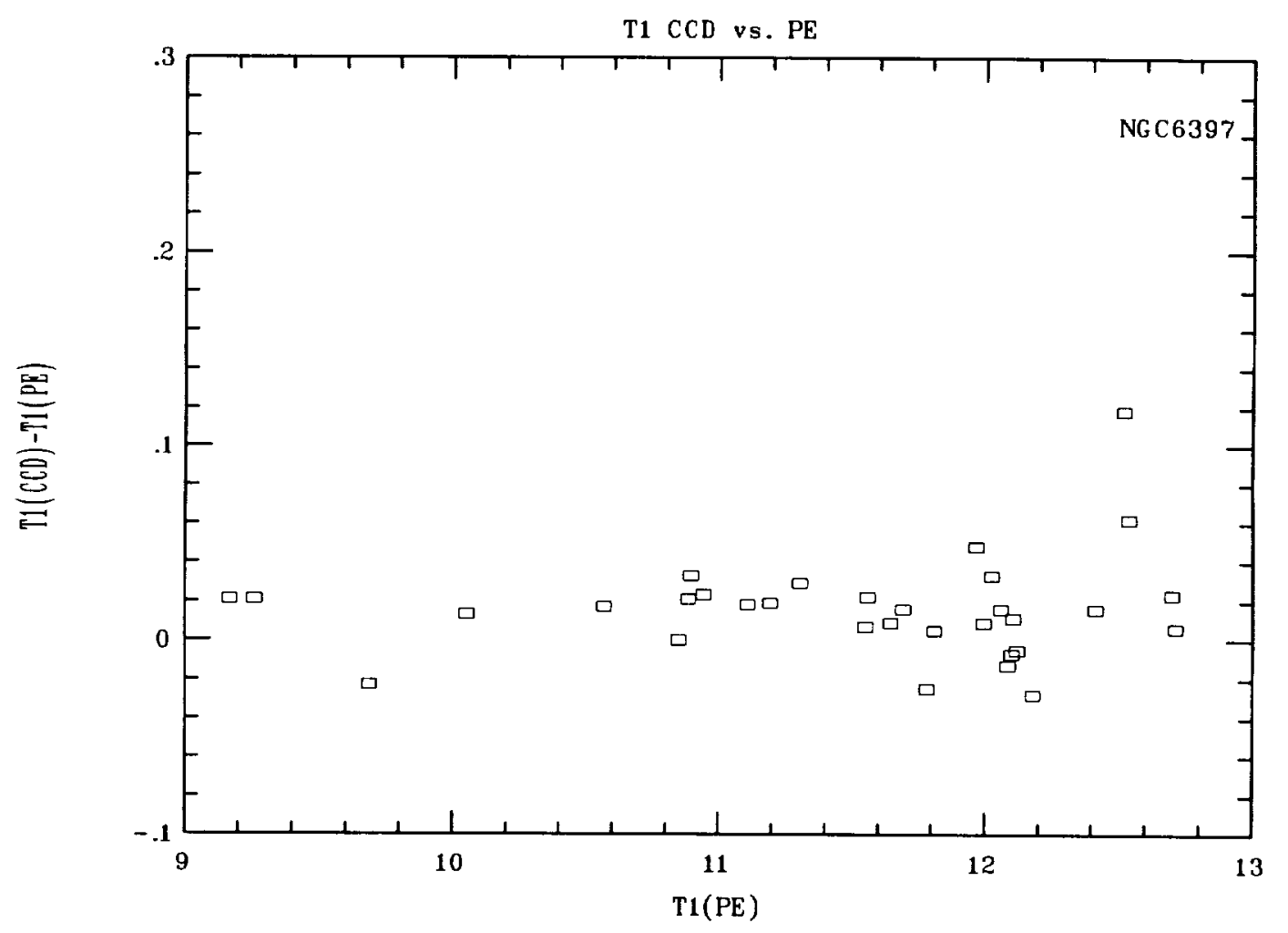

Fig. 16 

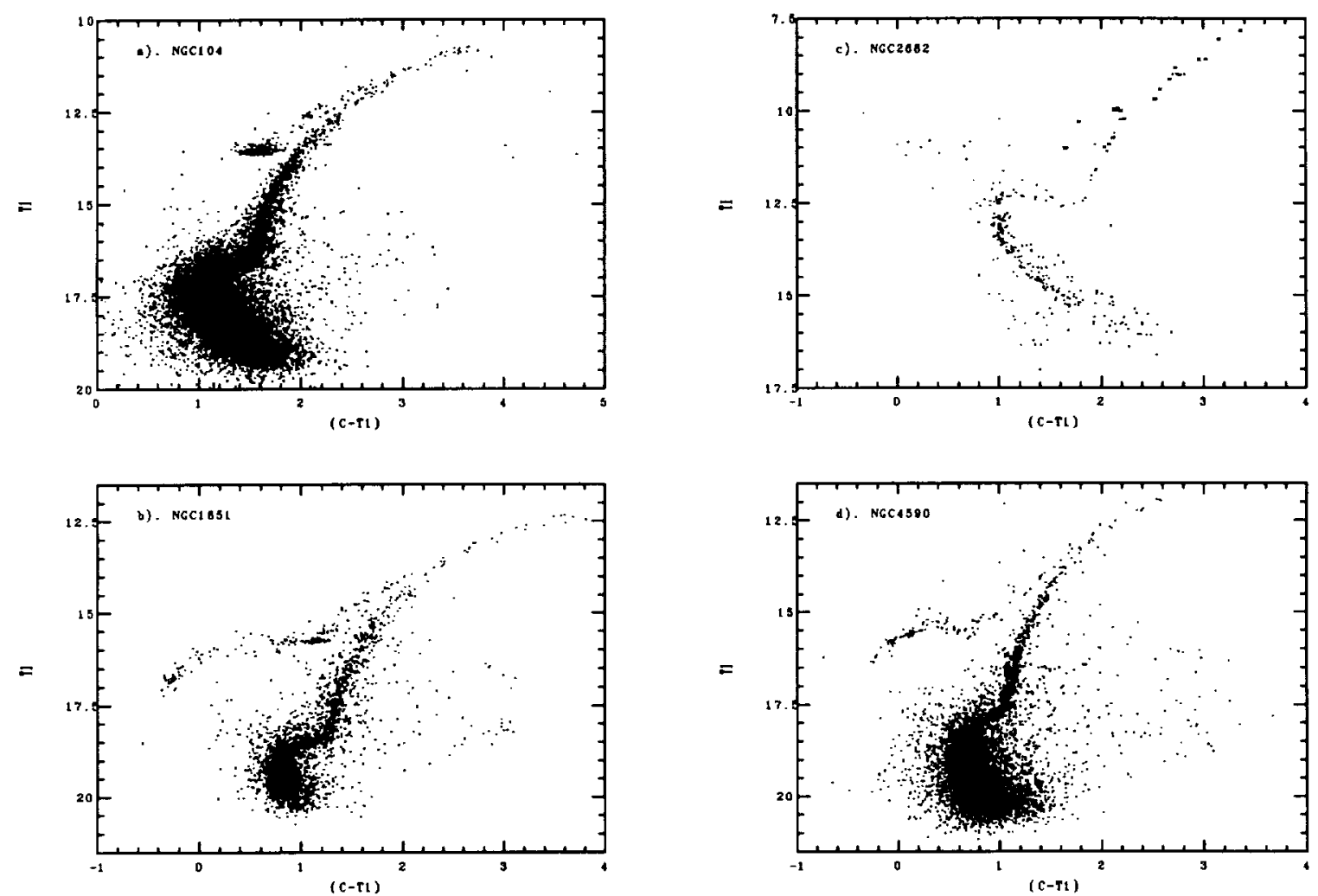

$F_{i j}, 2 a d$ 

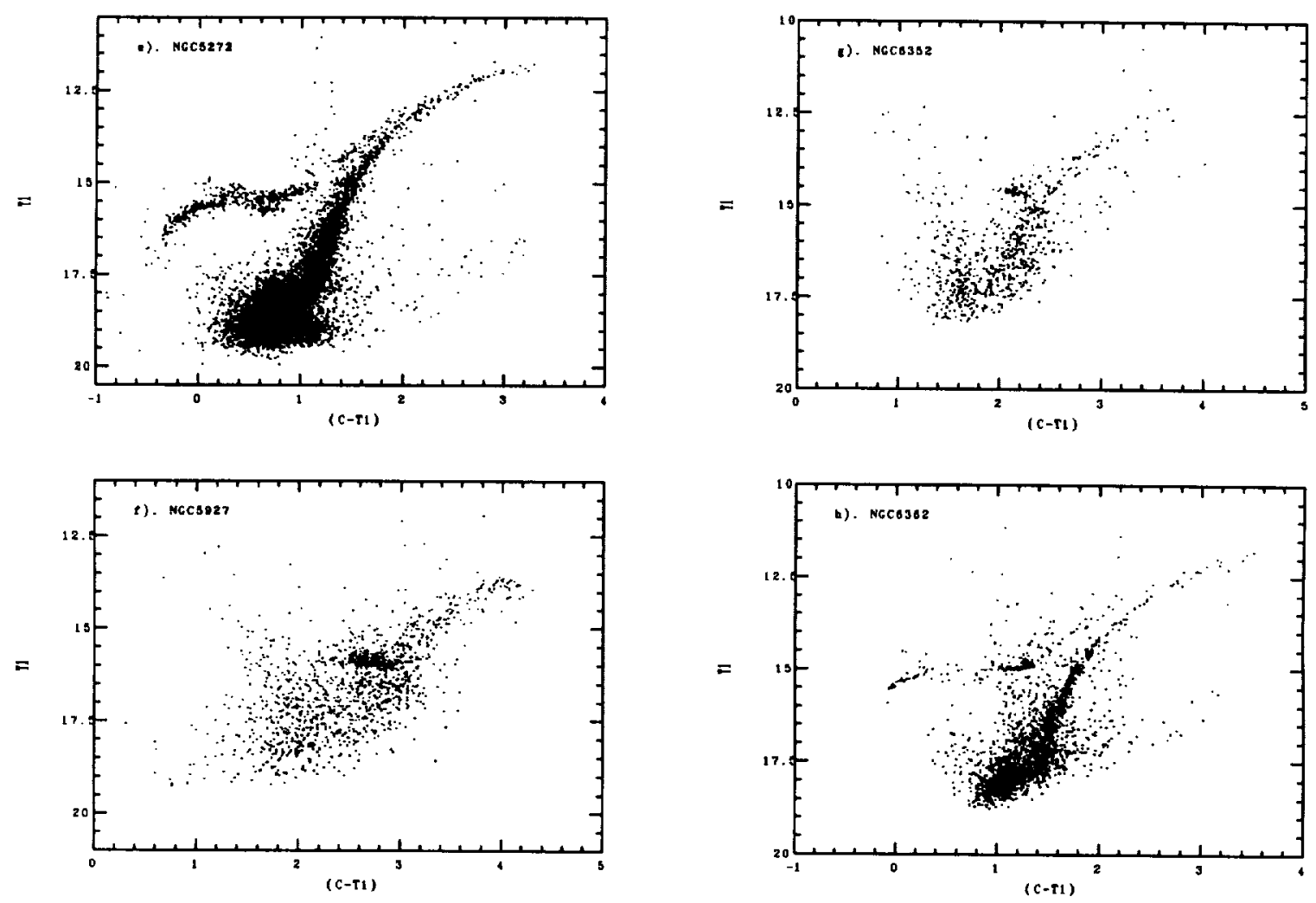

Fyzer 

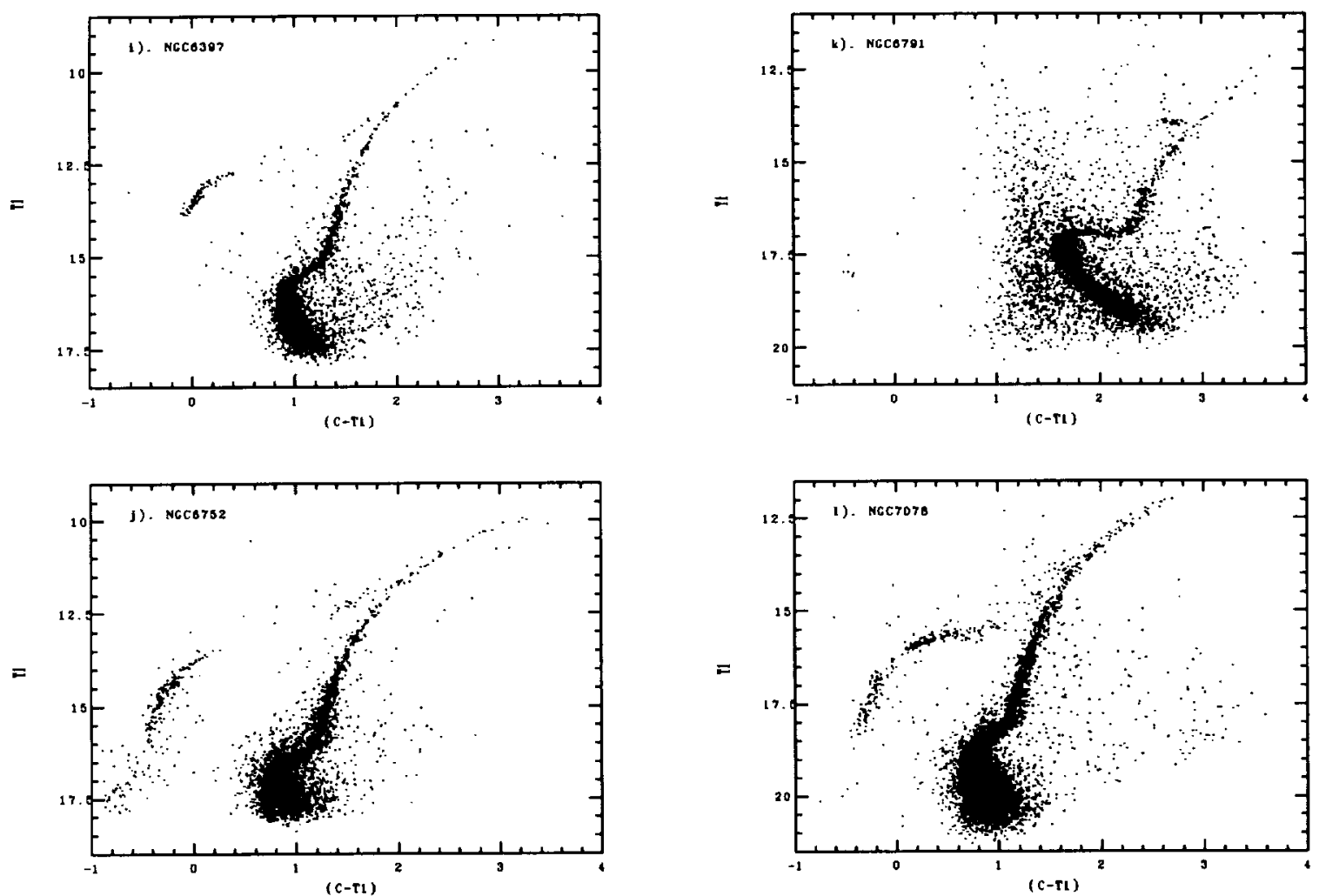

Fig. 2i-1 

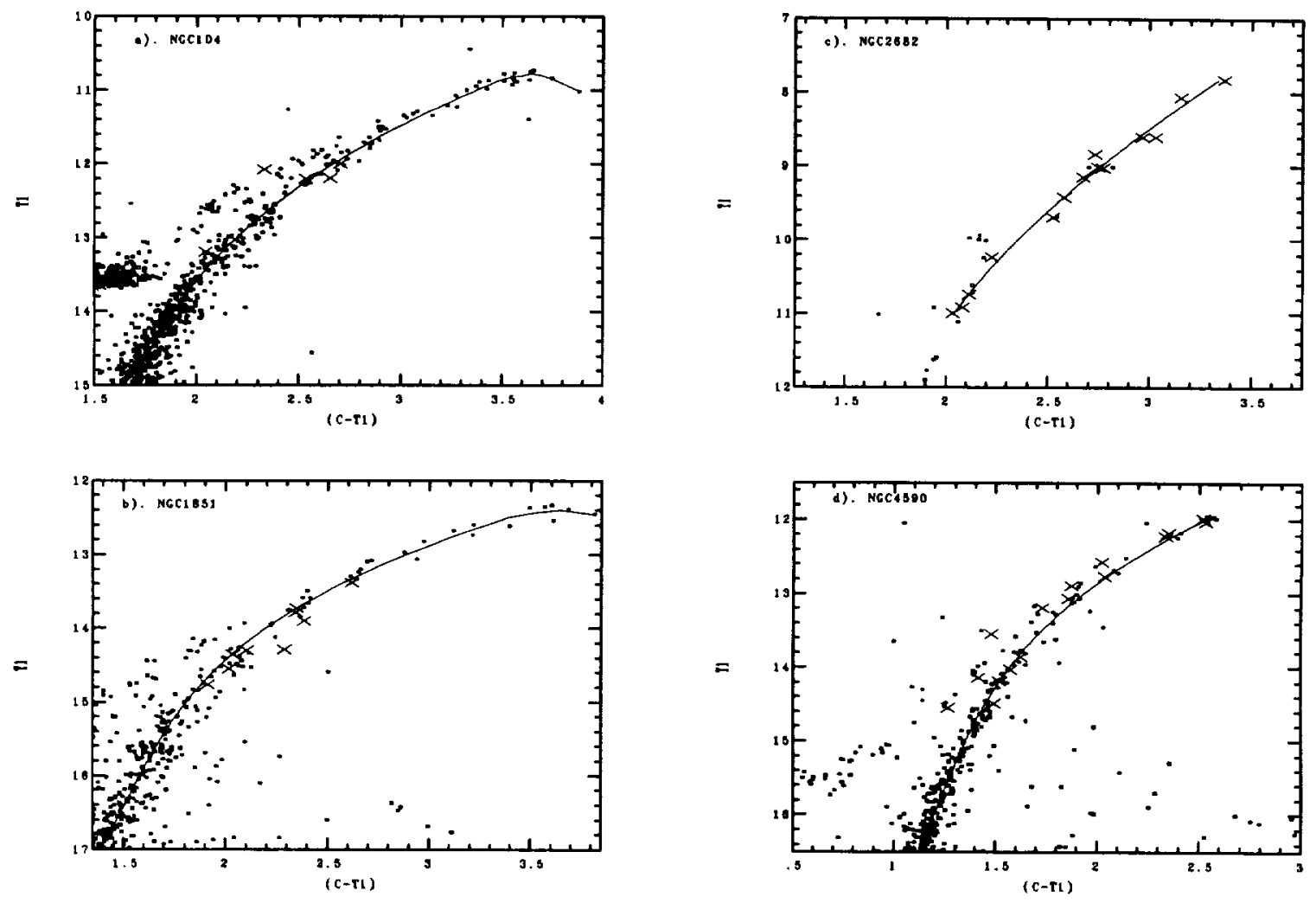

$F i g, 3 a-d$ 

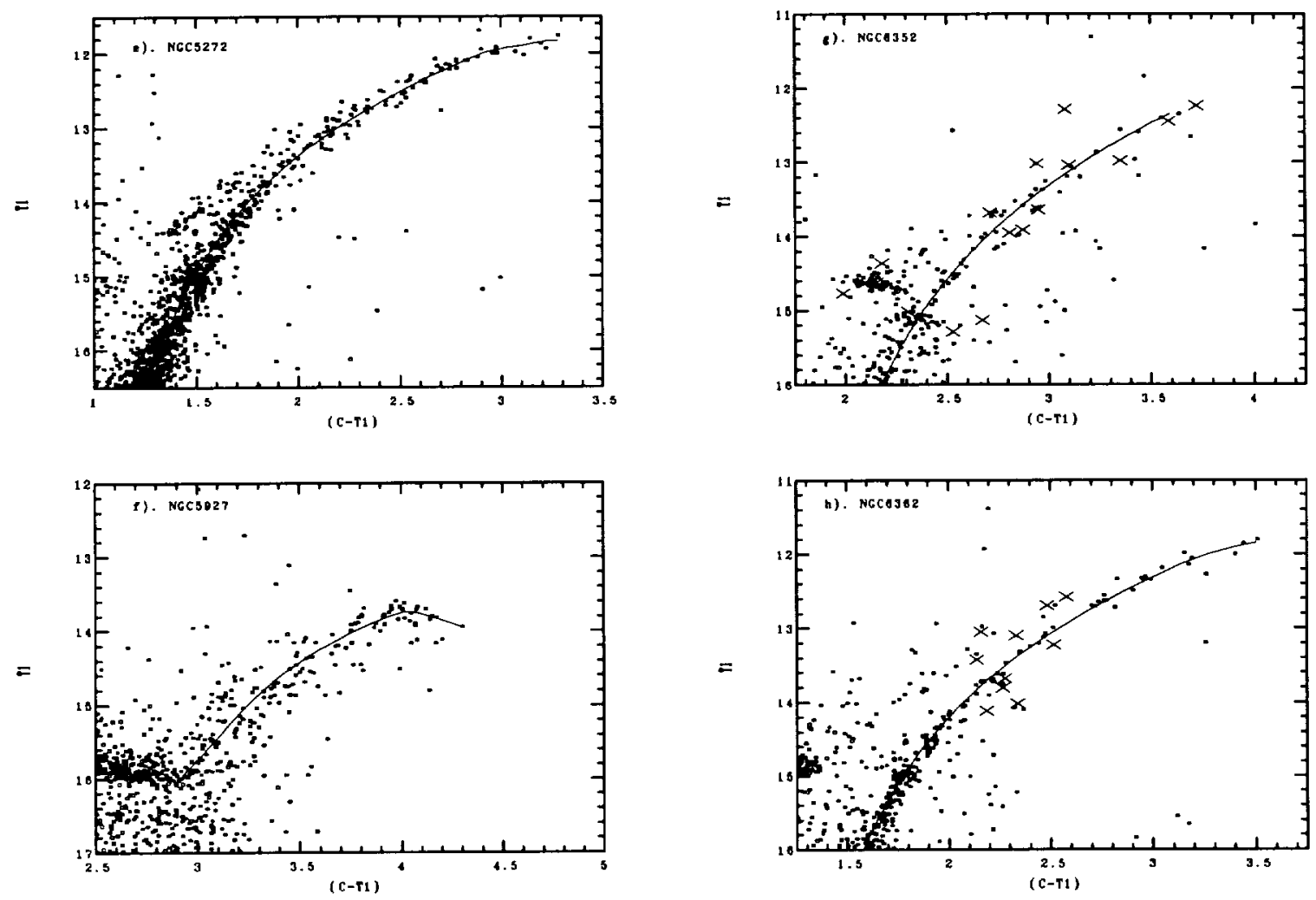

Fig $3 e-h$ 

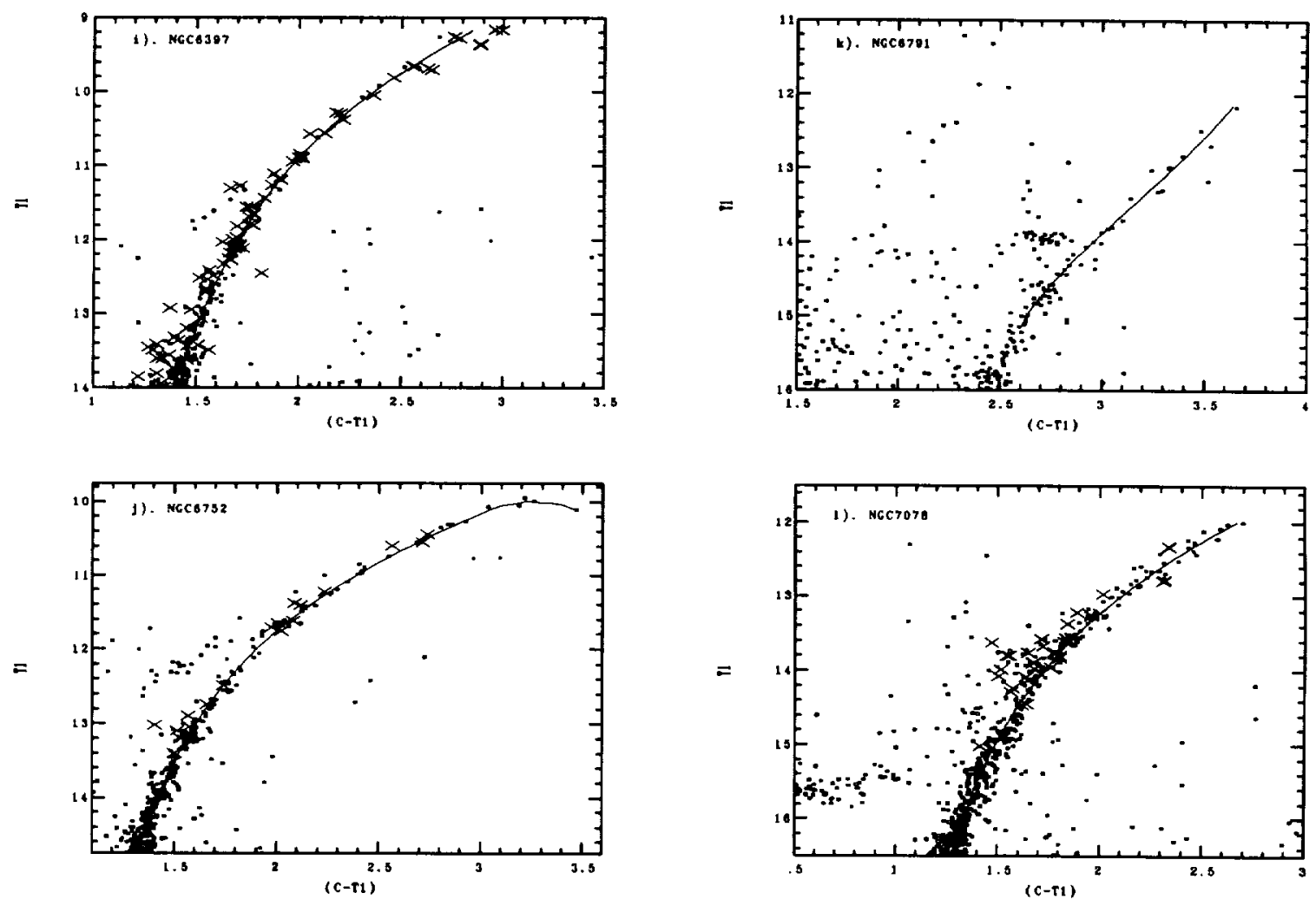

$$
F, 3 i-1
$$




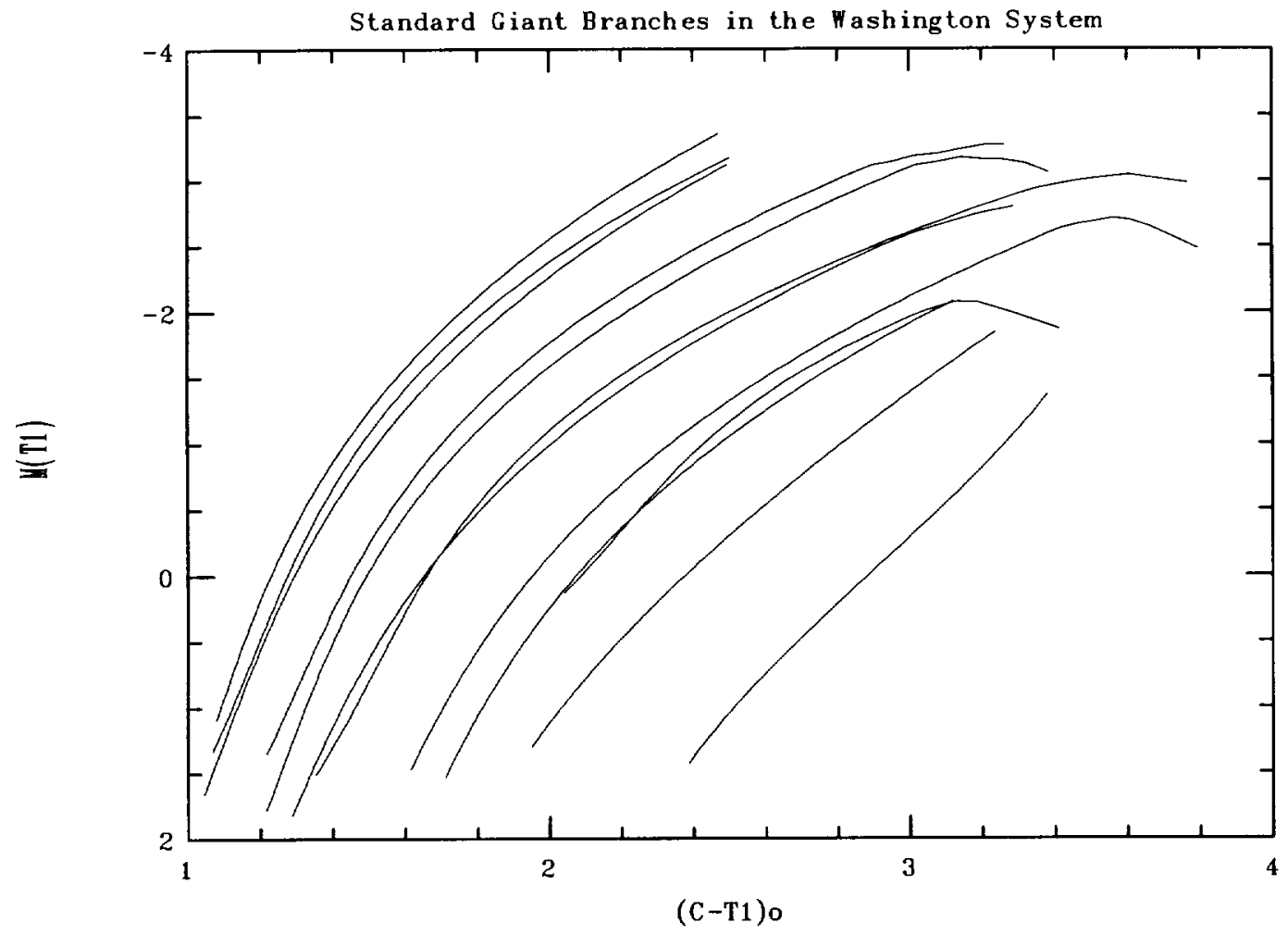

$F, 4$ 


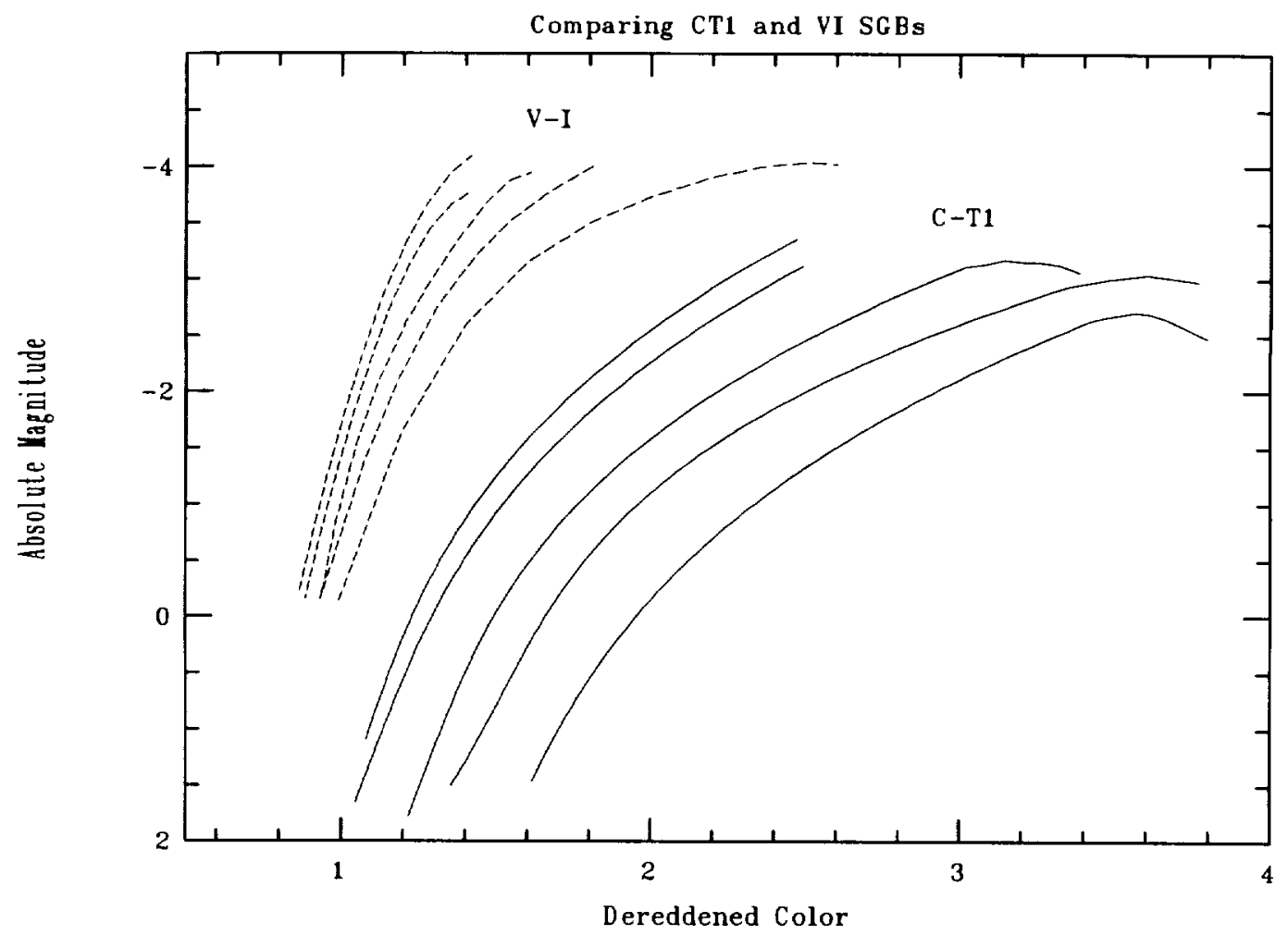

Fig. 5 

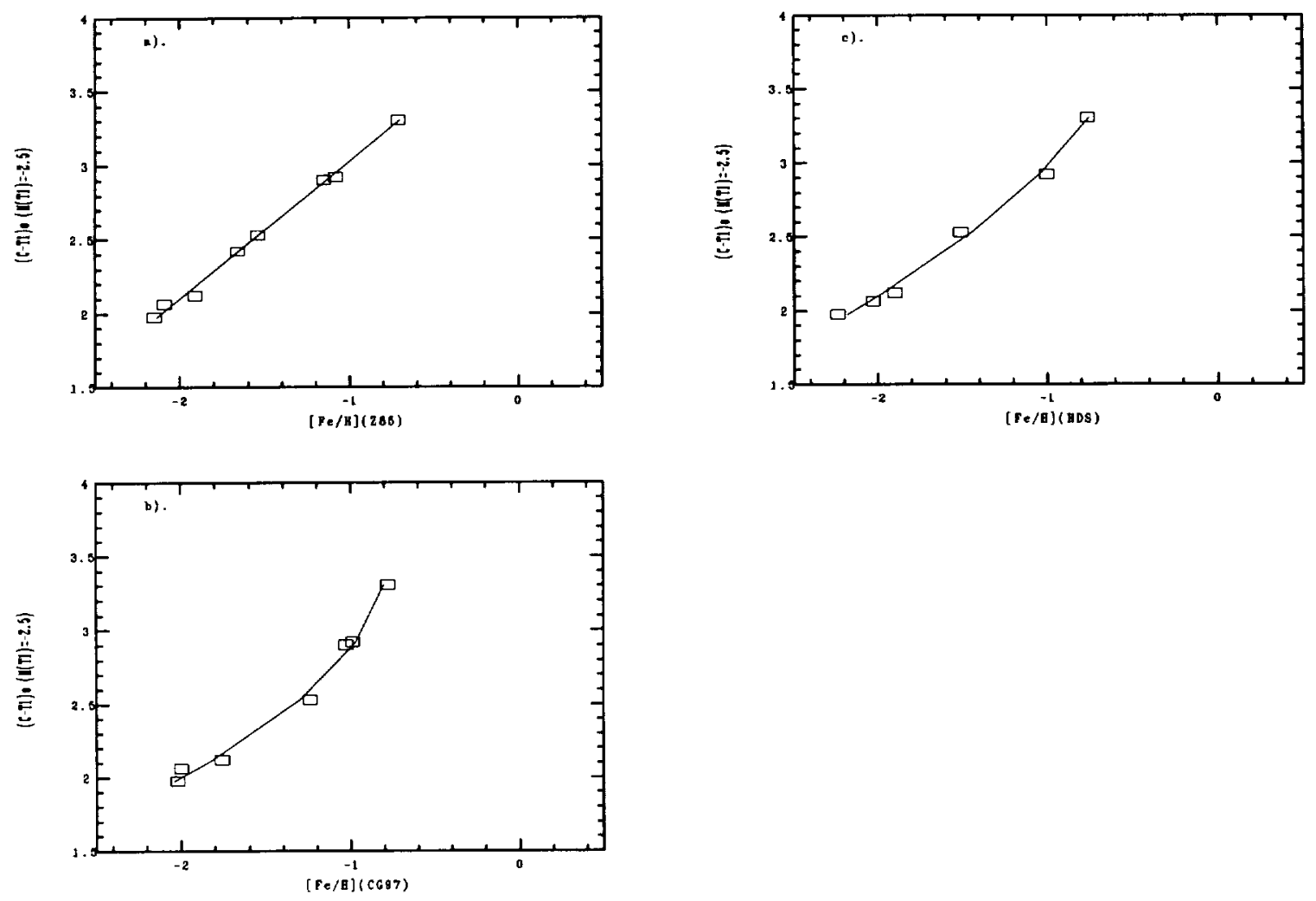

$F: 6 a-c$ 

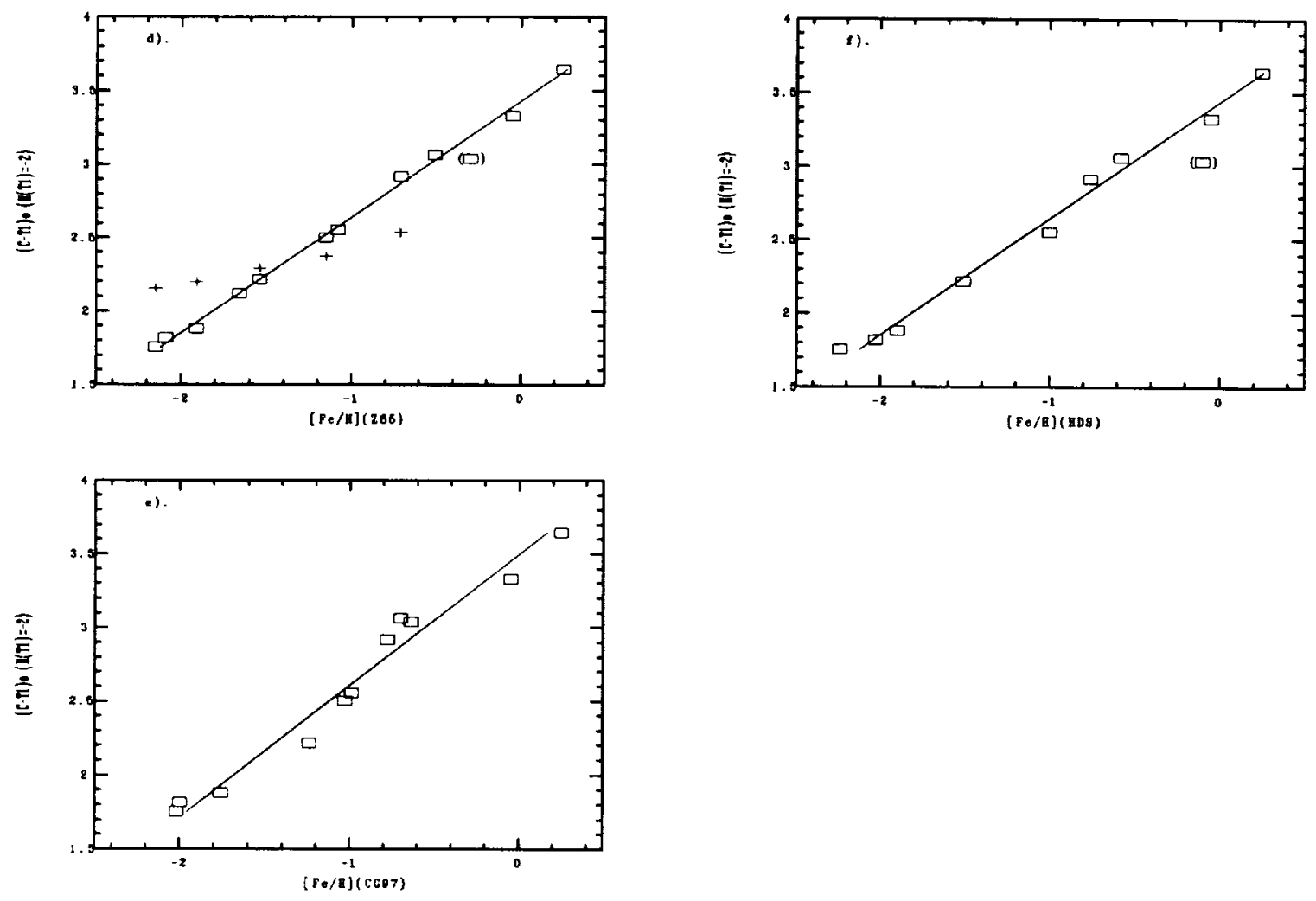

Fig.6d-f 

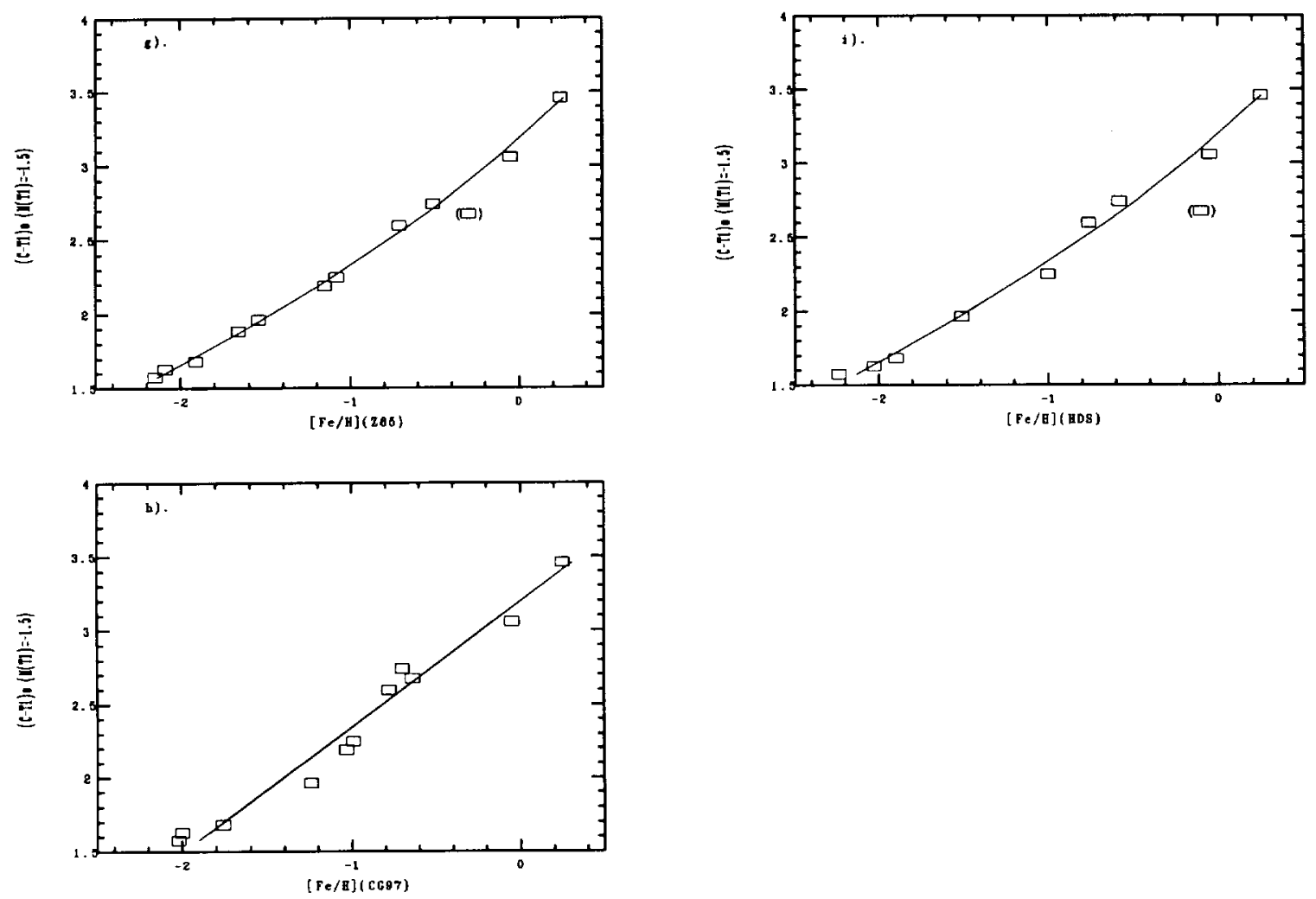

$F, \log -i$ 


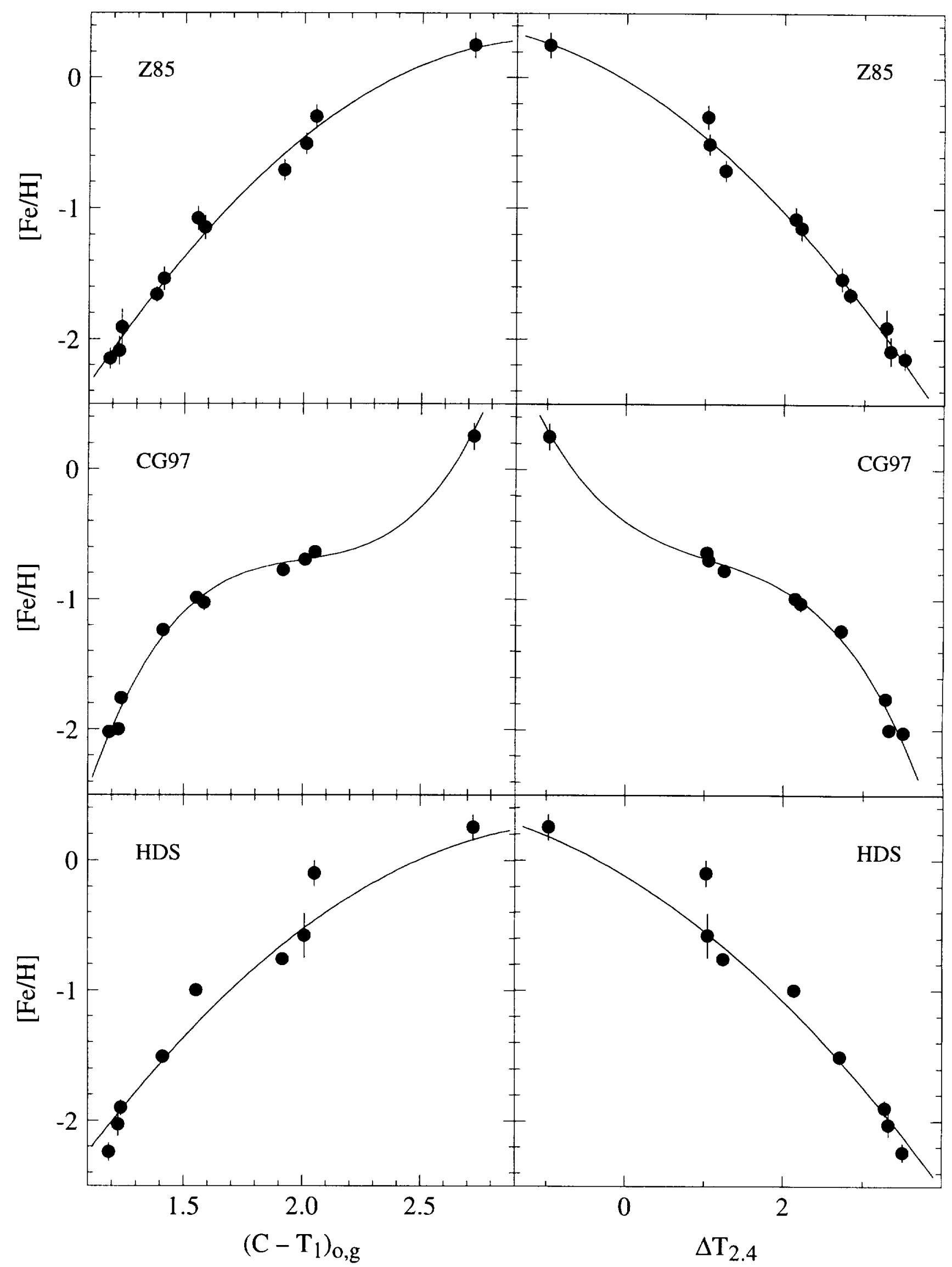

$F_{i g} . ?$ 


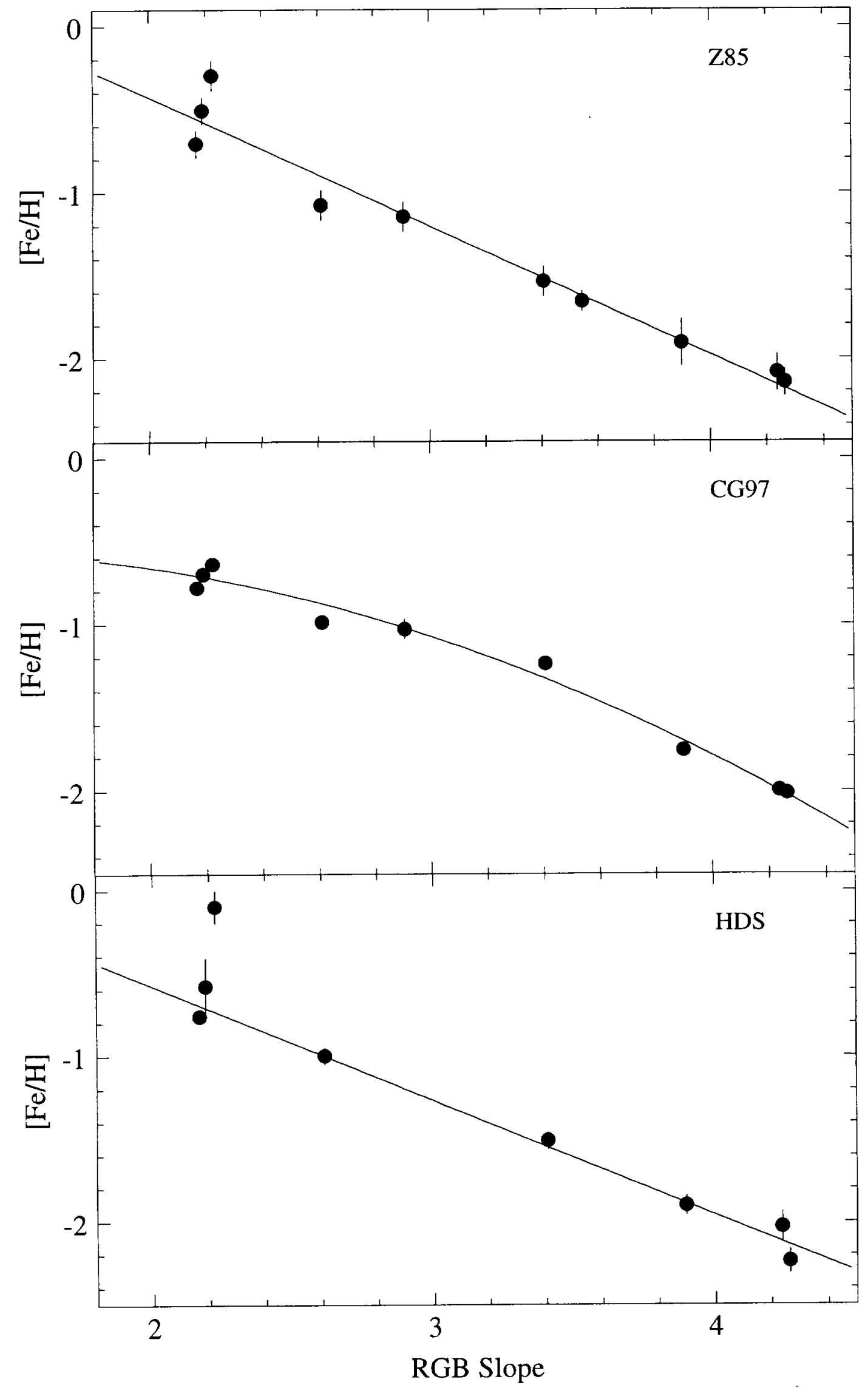

$F_{i j} .8$ 


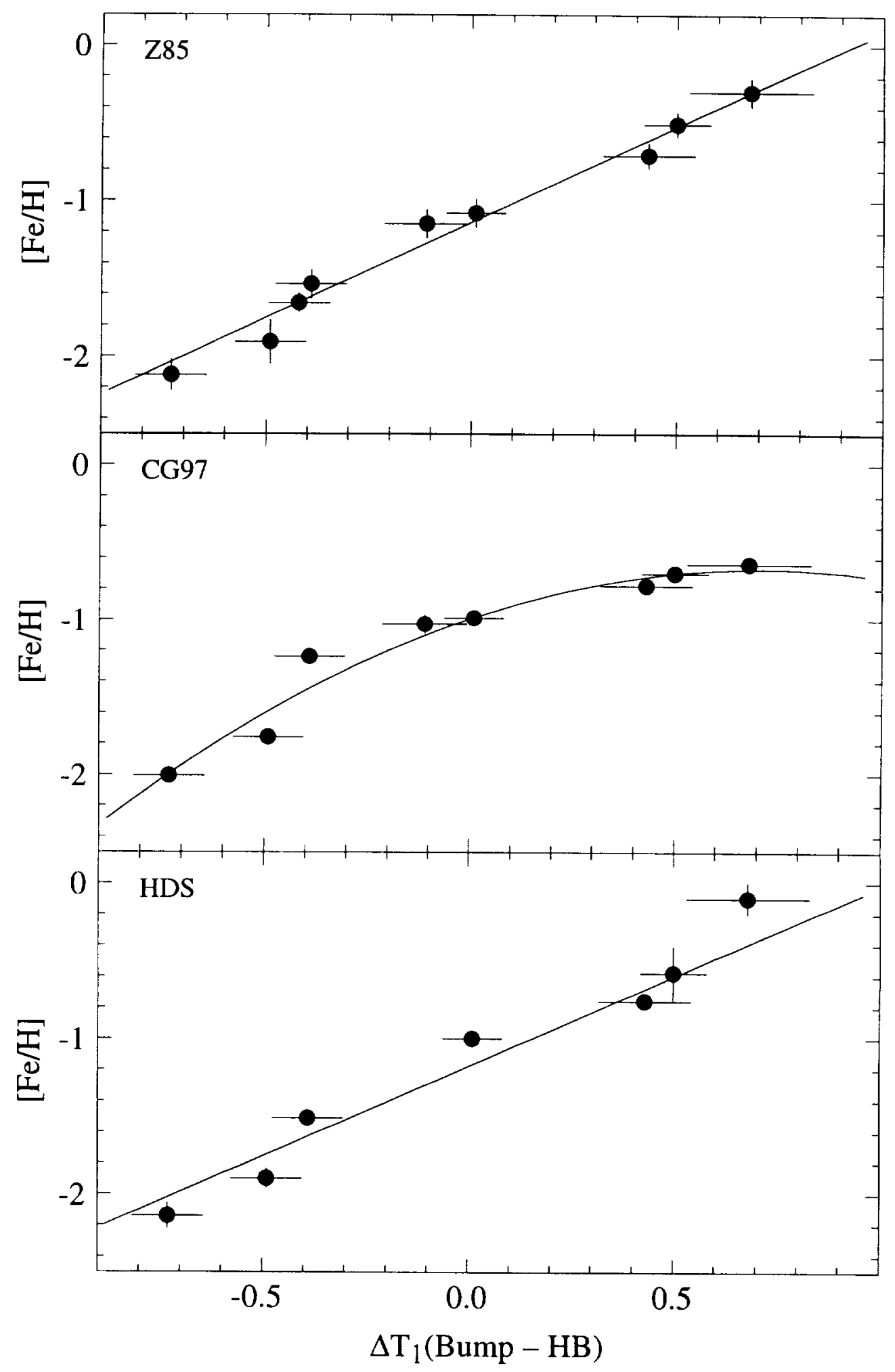

Fig. 9 


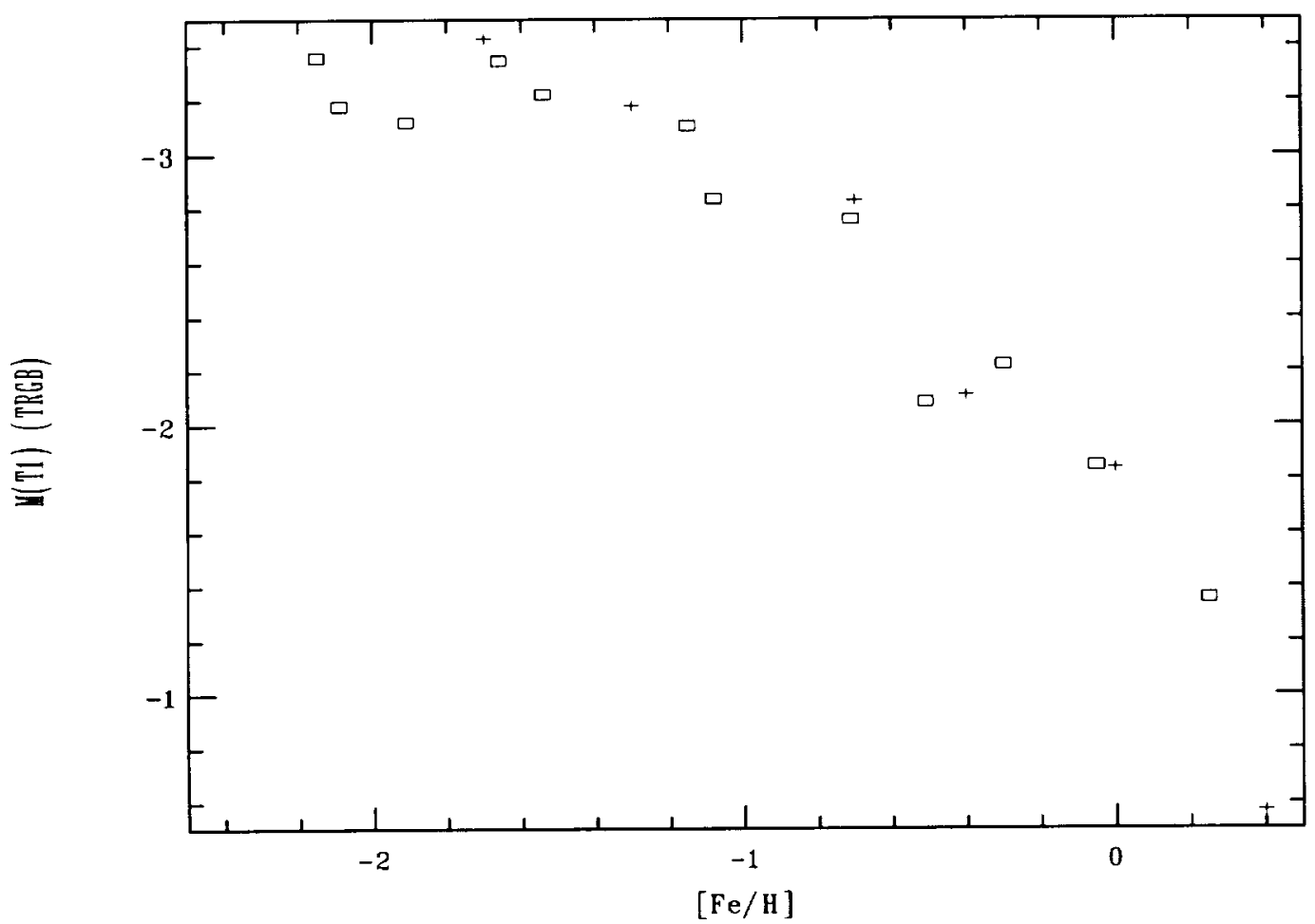

$F, 10$ 

TABLE 1

PARAMETERS FOR THE STANDARD CLUSTERS

\begin{tabular}{cccccc}
\hline NGC & $\mathrm{E}(\mathrm{B}-\mathrm{V})$ & $(m-\mathrm{M})_{V}$ & {$[\mathrm{Fe} / \mathrm{H}]_{Z 85}$} & {$[\mathrm{Fe} / \mathrm{H}]_{C G 97}$} & {$[\mathrm{Fe} / \mathrm{H}]_{H D S}$} \\
\hline $104(47 \mathrm{Tuc})$ & 0.04 & 13.51 & -0.71 & -0.78 & -0.76 \\
1851 & 0.02 & 15.45 & -1.15 & -1.03 & - \\
$2682(\mathrm{M} 67)$ & 0.05 & 9.70 & -0.05 & -0.05 & -0.05 \\
$4590(\mathrm{M} 68)$ & 0.03 & 15.14 & -2.09 & -2.00 & -2.03 \\
$5272(\mathrm{M} 3)$ & 0.01 & 15.11 & -1.66 & - & - \\
5927 & 0.45 & 16.08 & -0.30 & -0.64 & -0.1 \\
6352 & 0.24 & 14.57 & -0.51 & -0.70 & -0.58 \\
6362 & 0.11 & 14.70 & -1.08 & -0.99 & -1.00 \\
6397 & 0.18 & 12.40 & -1.91 & -1.76 & -1.90 \\
6752 & 0.04 & 13.19 & -1.54 & -1.24 & -1.51 \\
6791 & 0.13 & 13.60 & +0.25 & +0.25 & +0.25 \\
$7078(\mathrm{M} 15)$ & 0.10 & 15.41 & -2.15 & -2.02 & -2.24 \\
\hline
\end{tabular}


TABLE 2

COMPARISON OF CCD AND PHOTOELECTRIC DATA

\begin{tabular}{cccccccc}
\hline \hline NGC & $\Delta\left(C-T_{1}\right)$ & $\sigma$ & $\mathrm{N}$ & $\Delta\left(T_{1}\right)$ & $\sigma$ & $\mathrm{N}$ & Source \\
\hline 104 & -0.018 & - & 1 & -0.058 & - & 1 & 1 \\
1851 & 0.004 & 0.045 & 6 & -0.026 & 0.011 & 6 & 2 \\
2682 & 0.000 & 0.028 & 9 & 0.000 & 0.008 & 10 & 3,4 \\
4590 & 0.047 & 0.039 & 13 & -0.017 & 0.055 & 13 & 5,6 \\
5272 & -0.026 & 0.023 & 7 & - & - & - & 7 \\
6352 & 0.011 & 0.076 & 13 & -0.019 & 0.075 & 13 & 4,8 \\
6362 & 0.004 & 0.126 & 10 & -0.006 & 0.042 & 9 & 2 \\
6397 & 0.062 & 0.017 & 26 & 0.013 & 0.019 & 31 & 6,9 \\
6752 & 0.017 & 0.029 & 13 & 0.025 & 0.025 & 13 & 2 \\
6791 & -0.031 & 0.054 & 12 & - & - & - & 10 \\
7078 & 0.032 & 0.041 & 27 & 0.028 & 0.061 & 28 & 6,9 \\
& & & & & & & \\
\hline
\end{tabular}

References.--1. Geisler 1986a 2. Geisler et al. 1997 3. Canterna 1976a 4. Geisler et al. 1991 5. Minniti and Clariá 1989 6. Geisler et al. 1992 7. Canterna 1976b 8. Geisler 1986b 9. Clariá and Torres 199810. Canterna et al. 1986 
TABLE 3

POLYNOMIAL COEFFICIENTS

\begin{tabular}{cccccc}
\hline \hline NGC & $T_{1}$ Offset & $\mathrm{a}$ & $\mathrm{b}$ & $\mathrm{c}$ & $\mathrm{d}$ \\
\hline 104 & 12.5 & 2.408 & -0.470 & 0.0992 & -0.0107 \\
1851 & 15 & 1.808 & -0.284 & 0.0812 & -0.0233 \\
2682 & 10 & 2.350 & -0.361 & 0.0483 & 0.0028 \\
4590 & 15 & 1.353 & -0.172 & 0.0315 & -0.0137 \\
5272 & 15 & 1.489 & -0.205 & 0.0437 & -0.0151 \\
5927 & 15 & 3.243 & -0.364 & 0.0979 & -0.0690 \\
6352 & 15 & 2.383 & -0.252 & 0.0526 & -0.0093 \\
6362 & 15 & 1.773 & -0.236 & 0.0513 & -0.0115 \\
6397 & 12.5 & 1.610 & -0.171 & 0.0303 & -0.0091 \\
6752 & 12.5 & 1.736 & -0.293 & 0.0766 & -0.0125 \\
6791 & 14 & 2.953 & -0.380 & 0.0348 & 0.0220 \\
7078 & 15 & 1.486 & -0.189 & 0.0423 & -0.0084 \\
\hline
\end{tabular}


TABLE 4

STANDARD GIANT BRANCH POINTS

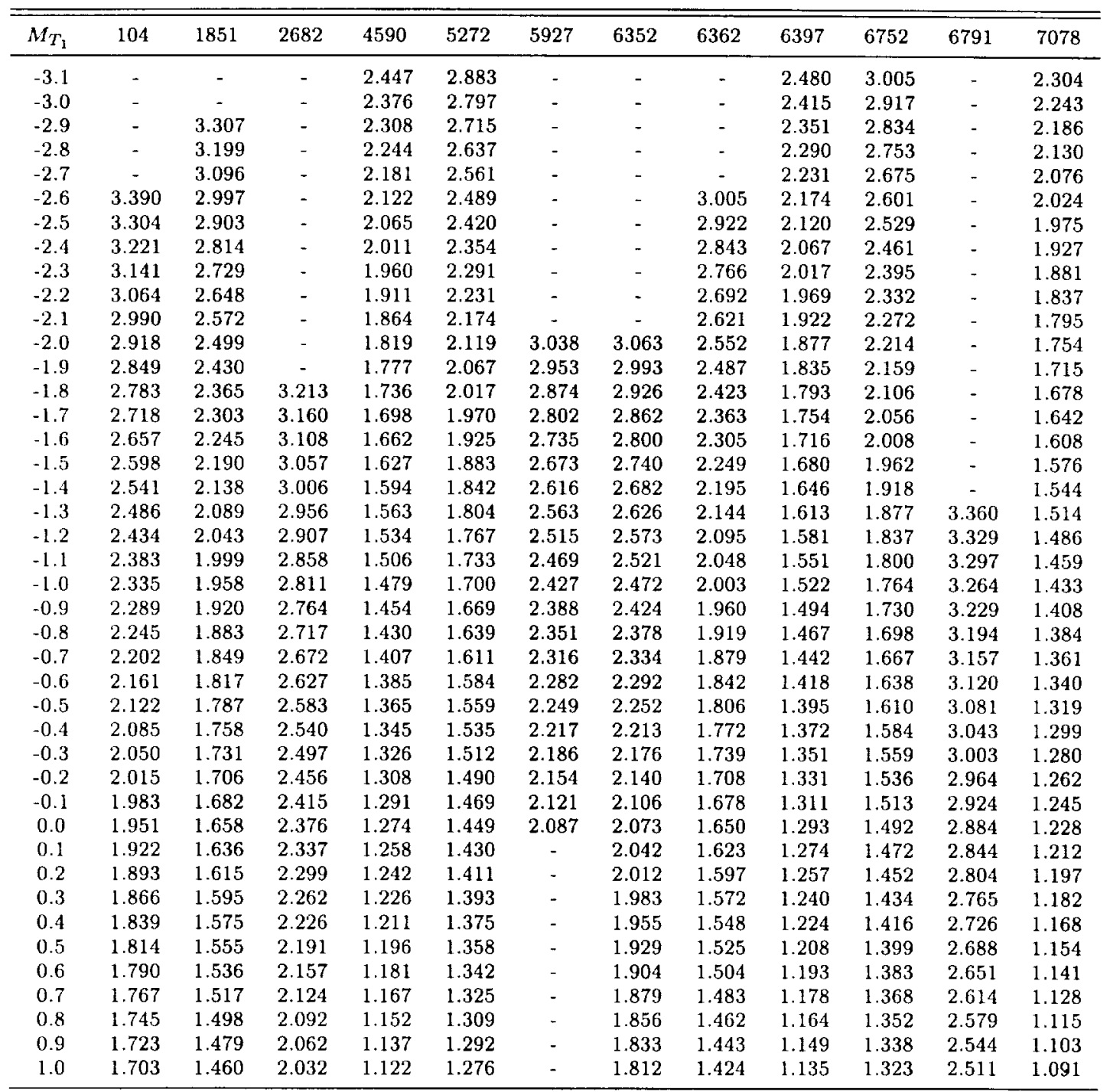


TABLE 5

ADDITIONAL STANDARD GIANT BRANCH POINTS

\begin{tabular}{|c|c|c|c|c|c|c|c|c|}
\hline Cluster & $M_{T_{1}}$ & $\left(C-T_{1}\right)_{0}$ & Cluster & $M_{T_{1}}$ & $\left(C-T_{1}\right)_{0}$ & Cluster & $M_{T_{1}}$ & $\left(C-T_{1}\right)_{0}$ \\
\hline 104 & $\begin{array}{l}-2.634 \\
-2.661 \\
-2.685 \\
-2.710 \\
-2.693 \\
-2.645 \\
-2.475\end{array}$ & $\begin{array}{l}3.420 \\
3.461 \\
3.515 \\
3.563 \\
3.610 \\
3.664 \\
3.795\end{array}$ & 1851 & $\begin{array}{l}-2.938 \\
-3.000 \\
-3.040 \\
-2.976\end{array}$ & $\begin{array}{l}3.350 \\
3.474 \\
3.604 \\
3.769\end{array}$ & 2682 & -1.846 & 3.237 \\
\hline 4590 & -3.173 & 2.500 & 5272 & $\begin{array}{l}-3.117 \\
-3.142 \\
-3.184 \\
-3.206 \\
-3.231 \\
-3.271 \\
-3.276\end{array}$ & $\begin{array}{l}2.897 \\
2.950 \\
3.014 \\
3.078 \\
3.132 \\
3.213 \\
3.263\end{array}$ & 5927 & $\begin{array}{l}-2.019 \\
-2.047 \\
-2.076 \\
-2.068 \\
-1.995 \\
-1.870\end{array}$ & $\begin{array}{l}3.055 \\
3.091 \\
3.126 \\
3.191 \\
3.280 \\
3.415\end{array}$ \\
\hline 6352 & -2.081 & 3.121 & 6362 & $\begin{array}{l}-2.628 \\
-2.689 \\
-2.737 \\
-2.794\end{array}$ & $\begin{array}{l}3.046 \\
3.119 \\
3.184 \\
3.289\end{array}$ & 6397 & -3.122 & 2.495 \\
\hline 6752 & $\begin{array}{l}-3.117 \\
-3.137 \\
-3.176 \\
-3.156 \\
-3.156 \\
-3.127 \\
-3.059\end{array}$ & $\begin{array}{l}3.020 \\
3.079 \\
3.143 \\
3.212 \\
3.257 \\
3.321 \\
3.386\end{array}$ & 6791 & -1.375 & 3.381 & 7078 & $\begin{array}{l}-3.200 \\
-3.300 \\
-3.362\end{array}$ & $\begin{array}{l}2.366 \\
2.430 \\
2.472\end{array}$ \\
\hline
\end{tabular}


TABLE 6

METALLICITY CALIBRATION COEFFICIENTS

\begin{tabular}{cccccc}
\hline \hline$\left(M_{T_{1}}\right.$, Met. scale $)$ & $\mathrm{a}$ & $\mathrm{b}$ & $\mathrm{c}$ & $\mathrm{N}$ & $\mathrm{RMS}$ \\
\hline$(-2.5$, Z85) & -4.273 & 1.081 & - & 8 & 0.03 \\
$(-2.5$, CG97) & -7.309 & 3.700 & -0.524 & 7 & 0.06 \\
$(-2.5$, HDS $)$ & -6.513 & 2.855 & -0.336 & 6 & 0.05 \\
$(-2.0$, Z85) & -4.350 & 1.269 & - & 11 & 0.04 \\
$(-2.0$, CG97) & -3.930 & 1.124 & - & 11 & 0.13 \\
$(-2.0$, HDS $)$ & -4.332 & 1.259 & - & 9 & 0.08 \\
$(-1.5$, Z85) & -5.297 & 2.335 & -0.210 & 11 & 0.04 \\
$(-1.5$, CG97) & -3.750 & 1.174 & - & 11 & 0.12 \\
$(-1.5$, HDS $)$ & -5.246 & 2.296 & -0.204 & 9 & 0.08 \\
\hline
\end{tabular}


TABLE 7

GIANT BRANCH DISPERSIONS

\begin{tabular}{rccc}
\hline \hline NGC & N & $\sigma\left(C-T_{1}\right)$ & Max. Metallicity Disp. (dex) \\
\hline 104 & 30 & 0.055 & 0.07 \\
1851 & 15 & 0.057 & 0.07 \\
4590 & 15 & 0.068 & 0.085 \\
5272 & 57 & 0.065 & 0.08 \\
5927 & 34 & 0.103 & 0.13 \\
6352 & 10 & 0.053 & 0.065 \\
6362 & 19 & 0.051 & 0.065 \\
6397 & 10 & 0.021 & 0.025 \\
6752 & 20 & 0.032 & 0.04 \\
7078 & 42 & 0.036 & 0.045 \\
\hline
\end{tabular}


TABLE 8

PARAMETERS For SRM, RGB SLOPE AND BUMP METHODS

\begin{tabular}{cccccc}
\hline NGC & $T_{1}(H B)$ & $\left(C-T_{1}\right)_{g}$ & $\Delta T_{2.4}$ & RGB Slope & $T_{1}($ Bump $)$ \\
\hline $104(47$ Tuc $)$ & $13.60 \pm 0.03$ & 1.996 & 1.25 & 2.17 & $14.03 \pm 0.10$ \\
1851 & $15.79 \pm 0.05$ & 1.624 & 2.21 & 2.91 & $15.68 \pm 0.09$ \\
$4590(\mathrm{M} 68)$ & $15.42 \pm 0.04$ & 1.286 & 3.33 & 4.24 & - \\
$5272(\mathrm{M} 3)$ & $15.46 \pm 0.07$ & 1.403 & 2.82 & 3.55 & $15.04 \pm 0.03$ \\
5927 & $15.92 \pm 0.03$ & 2.937 & 1.03 & 2.22 & $16.60 \pm 0.12$ \\
6352 & $14.64 \pm 0.03$ & 2.481 & 1.05 & 2.19 & $15.14 \pm 0.04$ \\
6362 & $15.01 \pm 0.04$ & 1.770 & 2.14 & 2.61 & $15.02 \pm 0.06$ \\
6397 & $12.60 \pm 0.07$ & 1.593 & 3.28 & 3.90 & $12.11 \pm 0.05$ \\
6752 & $13.58 \pm 0.07$ & 1.493 & 2.72 & 3.41 & $13.19 \pm 0.05$ \\
6791 & $13.93 \pm 0.03$ & 2.980 & -0.97 & - & - \\
$7078(\mathrm{M} 15)$ & $15.61 \pm 0.07$ & 1.385 & 3.51 & 4.26 & $14.88 \pm 0.05^{*}$ \\
\hline
\end{tabular}

* Derived by combining the NGC4590 and NGC7078 photometry. 
TABLE 9

Simultaneous Reddening and Metallicity coefficients

\begin{tabular}{ccccccc}
\hline \hline (Coefficient Subscript, Met. scale) & $\mathbf{a}$ & $\mathbf{b}$ & $\mathrm{c}$ & $\mathrm{d}$ & $\mathrm{N}$ & $\mathrm{RMS}$ \\
\hline$(1, \mathrm{Z85})$ & -6.226 & 4.299 & -0.7082 & - & 11 & 0.08 \\
$(2, \mathrm{Z} 85)$ & -0.0230 & -0.3619 & -0.07239 & - & 11 & 0.08 \\
$(1$, CG97) & -17.92 & 25.25 & -12.48 & 2.080 & 10 & 0.06 \\
$(2, \mathrm{CG} 97)$ & -0.4030 & -0.4293 & 0.2146 & -0.06550 & 10 & 0.07 \\
$(1$, HDS $)$ & -5.617 & 3.703 & -0.5812 & - & 9 & 0.18 \\
$(2$, HDS $)$ & -0.1181 & -0.3627 & -0.05872 & - & 9 & 0.17 \\
\hline
\end{tabular}


TABLE 10

RGB SLOPE COEFFICIENTS

\begin{tabular}{cccccc}
\hline \hline Metallicity Scale & $\mathrm{a}$ & $\mathrm{b}$ & $\mathrm{c}$ & $\mathrm{N}$ & $\mathrm{RMS}$ \\
\hline Z85 & 1.123 & -0.7760 & - & 10 & 0.13 \\
CG97 & -0.7391 & 0.3416 & -0.1511 & 9 & 0.06 \\
HDS & 0.7998 & -0.6897 & - & 8 & 0.24 \\
\hline
\end{tabular}


TABLE 11

RGB BUMP COEFFICIENTS

\begin{tabular}{cccccc}
\hline \hline Metallicity Scale & $\mathrm{a}$ & $\mathrm{b}$ & $\mathrm{c}$ & $\mathrm{N}$ & $\mathrm{RMS}$ \\
\hline Z85 & -1.139 & 1.230 & - & 9 & 0.09 \\
CG97 & -0.9961 & 0.9084 & -0.6372 & 8 & 0.10 \\
HDS & -1.180 & 1.155 & - & 7 & 0.16 \\
\hline
\end{tabular}


